\title{
A mathematically continuous model for describing the hydraulic properties of unsaturated porous media over the entire range of matric suctions
}

\author{
Yunquan Wang ${ }^{1 *}$, Jinzhu $\mathrm{Ma}^{2}$, Huade Guan ${ }^{3}$ \\ ${ }^{1}$ School of Environmental Studies, China University of Geosciences at Wuhan, 430074 \\ ${ }^{2}$ Key Laboratory of Western China's Environmental Systems (Ministry of Education), Lanzhou \\ University, 222 South Tianshui Road, Lanzhou 730000, China \\ ${ }^{3}$ School of the Environment and National Centre for Groundwater Research and Training, Flinders \\ University of South Australia, Australia
}

*Corresponding Author: Yunquan Wang, School of Environmental Studies, China University of Geosciences at Wuhan, Lumo Rd.388, Hongshan District, Wuhan, China, 430074 (wangyq@,cug.edu.cn)

\begin{abstract}
Recent studies suggest that water flow in unsaturated porous media extends beyond the commonly known capillary-driven regime into the film regime. There is a need to develop the unsaturated hydraulic properties over the entire range of matric suctions to capture both flow regimes. In this study, Fredlund and Xing model is modified to represent the soil water retention curve from saturation to oven dryness. The new function is mathematically differentiable. The hydraulic conductivity function is composed of the capillary-driven term and film associated term, which is easy to
\end{abstract}


apply. The new model has capacity to represent the bimodal hydraulic properties that are often present in structured and aggregated soils. Testing with the published data of sixteen soils shows good performance for both the water retention curve and the hydraulic conductivity function. For most soils, the new model results in a better agreement with observations than a published model. The result also indicates a possibility to improve the previously published film-associated hydraulic conductivity function.

Keywords: Soil hydraulic model; Film flow; Unsaturated porous media; Bimodal model

\section{Introduction}

The unsaturated zone supports terrestrial ecosystems. It lies between surface and groundwater systems, influencing groundwater recharge and quality. Thus modeling water flow and solute transport in the unsaturated zone is always an important research subject in hydrological and ecological studies. Solutions to the Richards' (1931) equation, the most widely used model for simulating water flow and associated solute transport in variably saturated media, require the soil water retention curve (SWRC) $h(\theta)$ and the hydraulic conductivity curve $K(h)$.

The commonly used SWRC functions (e.g., Brooks and Corey, 1964; van Genuchten, 1980) and soil hydraulic conductivity functions (e.g., Mualem, 1976a) are based on the capillary theory (Mualem, 1976a; Tuller and Or, 2001). This theory conceptualizes pore space into a bundle of cylindrical capillaries (Millington and 
Quirk, 1961; Mualem, 1976a). Water is supposed to move only when the capillary pendular rings are continuous in the pores (Tokunaga, 2009), with an assumption that the meniscus of water is axially symmetric or nearly so (Orr et al., 1975; Bear et al., 2011). The water content at the stage with broken pendular rings is defined as the residual water content $\left(\theta_{r}\right)$, representing the moisture beyond the capillary influence. This water is assumed to be hydraulically immobile according to the capillary theory. However, previous studies have found that the conventional SWRCs and soil hydraulic conductivity functions often do not accurately simulate flow processes that occur under conditions of low soil water contents (e.g., Rossi and Nimmo, 1994; Silva and Grifoll, 2007). Water moving along thin liquid films on the particle surfaces can be significant under dry conditions (Li and Wardlaw, 1986; Lenormand, 1990; Toledo et al., 1990; Tuller and Or, 2001; Wang et al., 2013), which is, however, neglected in the commonly used soil hydraulic models (Lebeau and Konrad, 2010). The impact from three-dimensional pore network (e.g., pore connectivity and air entrapment) further complicates unsaturated water flow modeling, which is not considered here.

To describe the soil hydraulic properties from saturation to oven dryness, one needs to extend the soil hydraulic models from the capillary-dominated suctions to film-dominated suction range.

Depending whether the van der Waals forces or the electrical double-layer forces are the dominant contribution to film thickness, two hydraulic conductivity models were developed to simulate film flow by Tuller and Or (2001) and Tokunaga (2009), respectively. The model proposed by Tuller and Or (2001) accounts for both capillary 
and film forces. This model must be used in conjunction with the water retention model of Or and Tuller (1999), which often fails to fit experimental data in the intermediate saturation range (Lebeau and Konrad, 2010). Additionally, the electrical double-layer force is considered more important at the intermediate values of matric potential (Tokunaga, 2011). By combining an existing capillary conductivity model (e.g., van Genuchten (1980) model) with Tokunaga (2009) film model that accounts for electrical double-layer forces, a series of models have been developed to describe the soil hydraulic conductivity from saturation to oven dryness (Lebeau and Konrad, 2010; Zhang, 2011; Peters, 2013). Two problems exist in these models. First, the film conductivity model presented by Tokunaga (2009) relies on the smooth sphere grain assumption, its validation for different soils needs to be examined. Secondly, for the SWRCs, these models all have the "mathematical discontinuity" problem (Iden and Duner, 2014). This problem results from that the water content is divided into the capillary and film components. The SWRCs that account for film flow (e.g., Campbell and Shiozawa (1992) model) are not differentiable over the entire range of suctions. This can lead to a numerical problem (Iden and Duner, 2014). A smoothing strategy may solve this problem, but it makes the model mathematically more complex.

Fredlund and Xing (1994) adapted a different approach. They describe the SWRC from saturation to oven dryness without partitioning water into the capillary and adsorptive components, so the equation is differentiable over the entire matric suction range. However, there is no closed-form solution for the hydraulic conductivity 
function, and film flow contribution is not considered.

In this paper, a modification of Fredlund and Xing (1994) model is provided to describe the SWRC over the suction range from saturation to oven dryness. The model is mathematically continuous and easy to use. Based on the modified model, an approximately closed-form function is derived to express the hydraulic conductivity that accounts for capillary force. The total conductivity is then described by combining the capillary conductivity with a conductivity model that accounts for film flow. The new model is tested with published data ranging from saturation to very dry conditions. Model performance is evaluated by comparing the results with an existing model.

\section{Model Development}

\subsection{Soil Water Retention Curve}

Fredlund and Xing (1994) proposed a model (hereafter, the FX model) to describe the soil water retention from saturation to zero water content, expressed as

$$
S=C(h)\left(\ln \left(e+|\alpha h|^{n}\right)\right)^{-m}
$$

where $S=\theta / \theta_{s}$ is the saturation degree, $\theta\left[\mathrm{L}^{3} \mathrm{~L}^{-3}\right]$ is the volumetric water content, $\theta_{s}$ is the saturated water content, $e$ is Euler's number (the $e$ constant), $h(\mathrm{~L})$ is the matric suction and $\alpha\left(\mathrm{L}^{-1}\right), n, m$ are all fitted parameters, $C(h)$ is a correction function defined as

$$
C(h)=1-\frac{\ln \left(1+h / h_{r}\right)}{\ln \left(1+h_{0} / h_{r}\right)}
$$

where $h_{r}$ is the matric suction corresponding to the residual water content, $\theta_{r}$, and the 
$h_{0}$ is the matric suction where water content is zero. Experimental data of different soils have previously shown that the average value of this matric suction is approximately $6.3 \times 10^{6} \mathrm{~cm}$ (Schneider and Goss, 2012), which yields a better representation of the oven dryness condition $\left(105^{\circ} \mathrm{C}\right)$ in comparison with the commonly used value of $10^{7} \mathrm{~cm}$ (Schneider and Goss, 2012). The correction function $C(h)$ is to make the water content decrease to zero at the suction of $h_{0}$.

Since $C(h)$ is also a function of $h$, it is difficult to obtain the $h(S)$ expression and to derive the capillary conductivity function. The flow based on capillary tubes accounts for water movement when soil water content is higher than $\theta_{r}$. To derive the capillary conductivity function, it only requires $h(S)$ expression in a range from saturation to $\theta_{r}$. This can be easily solved by introducing a slightly different correction function $\Gamma(h)$, defined as,

$$
\Gamma(h)=1-\frac{\ln \left[1+c h / h_{r}\right]}{\ln \left[1+c h_{0} / h_{r}\right]}
$$

Here, the parameter, $c$, is introduced to make sure that $\Gamma(h)$ is approximately equal to 1 for suctions smaller than $h_{r}$. For this purpose, $c$ should have a small value, however, it cannot be too small to make $\Gamma(h)$ only function on the matric suctions closing to $h_{0}$. Practically, $c$ is better to be in the magnitude of $10^{-2}$. Since the model performance doesn't change sensitively to the value of $c$, for simplicity, $c$ is suggested to be kept constant as 0.01 . Then, the saturation degree $S$ can be written as

$$
S(h)=\Gamma(h)\left(\ln \left(e+|\alpha h|^{n}\right)\right)^{-m}
$$

As discussed previously, the impact of $\Gamma(h)$ on the water content is negligible $(\Gamma(h)$ $\approx 1$ ) for suctions less than $h_{r}$. Then, the $h(S)$ expression can be easily derived as 


$$
h(S)=\frac{1}{\alpha}\left(e^{S^{-1 / m}}-e\right)^{1 / n}
$$

The difference between equations (1) and (4) is shown in Figure 1. In comparison to the FX model, the new model produces a larger saturation degree for suctions beyond $h_{r}$ than the model without the correction factor (equation (4) with $\Gamma(h)=1$ ), while it estimates the same values for suctions ranging from zero to $h_{r}$.

It is interesting that when $m$ has a small value, the new SWRC model yields a bimodal structure as shown in figure 1 a) and c). The bimodal pore-size distribution has been reported for structured or aggregated soils (Dexter and Richard, 2009; Romano et al., 2011). Thus, with a small $m$, the new model appears to be suitable for soils with bimodal SWRC. Two data sets illustrated here are soils 13 and 15 as shown in the results section.

\section{(Figure 1 near here)}

\subsection{Soil Hydraulic Conductivity Model}

Equation (4) describes the soil water retention function continuously from capillary-dominated to film-dominated suction ranges. The soil hydraulic conductivity, however, has to be separated into the capillary tube based flow and the film flow, due to their different physical mechanisms.

\subsubsection{Capillary Conductivity Model}

The specific volumetric flux (the volumetric flow rate per unit area of porous media) associated with the capillary force, $Q_{c}$, can be expressed as 


$$
Q_{c}=K_{c} \frac{d h}{d x}
$$

where $K_{c}$ is the capillary conductivity, $d h / d x$ is the matric suction gradient.

Capillary conductivity models assume a bundle of straight parallel tubes of different diameters to represent the pore system. The tubes are either fully saturated or empty. The contribution of fully saturated tubes to moisture content is derived from the SWRC and the velocity in saturated tube is calculated by the Hagen-Poiseuille equation. The bulk hydraulic conductivity is then determined by integrating the contributions of all saturated tubes at the specific water potential. A tortuosity factor is often introduced to account for the limitation of the straight parallel tubes assumption to represent real pores. The general form of such a model is written as (Hoffmann-Riem et al., 1999):

$$
K_{c}=K_{s} S_{c}^{l}\left[\int_{0}^{S_{c}} h^{-\kappa} d S_{c} / \int_{0}^{1} h^{-\kappa} d S_{c}\right]^{\beta}
$$

where $K_{s}$ is the hydraulic conductivity at saturation, $S_{c}$ is the effective saturation where the capillary force dominates. The three parameters $l, \kappa$ and $\beta$ vary in different models. In Burdine (1953) model, $l=2, \kappa=2$, and $\beta=1$, while in the model of Alexander and Skaggs (1986) $l=1, \kappa=1$, and $\beta=1$. When $l=0.5, \kappa=1$ and $\beta=2$, the model is equivalent to the Mualem (1976) model. The Mualem (1976) model remains the commonly used model. It has been shown to provide a reasonable description of the capillary conductivity. Thus, the Mualem model for capillary conductivity is adopted in this study. The parameter $l$ that accounts for tortuosity and connectivity is treated as a free fitting parameter as suggested in previous studies (e.g., Schaap and 
Leij, 2000; Peters, 2013).

The effective saturation, $S_{c}$, is defined as

$$
S_{c}=\frac{\theta-\theta_{r}}{\theta_{s}-\theta_{r}}=\frac{S-S_{r}}{1-S_{r}}
$$

where $S_{r}=\theta_{r} / \theta_{s}$, is the saturation degree at the residual water content. When $\theta$ is higher than $\theta_{r}$, substitution of equation (5) into equation (7) gives

$$
K_{c}=K_{s} S_{c}^{l}\left[\frac{f(S)}{f(1)}\right]^{2}
$$

where

$$
f(S)=\int_{S_{r}}^{S}\left(e^{S^{-1 / m}}-e\right)^{-1 / n} d S
$$

and

$$
f(1)=\int_{S_{r}}^{1}\left(e^{S^{-1 / m}}-e\right)^{-1 / n} d S
$$

Substitution of $S=y^{m}$ into equation (10) leads to

$$
f(y)=m \int_{S_{r}^{1 / m}}^{S^{1 / m}}\left(e^{1 / y}-e\right)^{-1 / n} y^{m-1} d y
$$

In its most general case, there is no closed-form expression for equation (12). Solving equation (12) using numerical integration would lead to numerical complexity. Equation (9) includes two scaling factors for the hydraulic conductivity, the power function of the effective saturation, $S_{c}^{l}$, and the integration part described in equations (10) and (11). As described previously, different values of parameters $\kappa$ and $\beta$ for the integration part have been used in different models. All of these models have shown acceptable representation of the observed soil hydraulic conductivity data (Burdine, 1953; Mualem, 1976a; Alexander and Skaggs, 1986). This may indicate that the difference between the integration parts can be complemented by the power function 
of the effective saturation, with different values of $l$.

The new SWRC (equation (4)) is in a similar form to the van Genucthen model (van Genucthen, 1980, hereafter the VG model). Here, the integrand function of the VG model is used to replace that of the new model, with an assumption that the difference is complemented by $S_{c}$. Then, the capillary conductivity (equation (9)) can be written as (see Appendix A)

$$
K_{c}=K_{s} S_{c}^{l}\left[\frac{\int_{S_{r}^{1 / m}}^{S^{1 / m}}(1-y)^{-1 / n} d y}{\int_{S_{r}{ }^{1 / m}}^{1}(1-y)^{-1 / n} d y}\right]^{2}=K_{s} S_{c}^{l}\left[1-\left(\frac{1-S^{1 / m}}{1-S_{r}^{1 / m}}\right)^{1-1 / n}\right]^{2}
$$

For water content less than $\theta_{r}, K_{c}$ equals to zero. Equation (13), with a simple form, has good capability to represent the unsaturated hydraulic conductivity for different soils as shown later.

\subsubsection{Hydraulic Conductivity for Film Flow}

The film velocity normal to film cross section is obtained by solving the simplified Navier-Stokes equation. Integrating the velocity distribution over film thickness and dividing by film thickness yield the averaged film velocity, expressed as (Bird et al., 1960)

$$
v_{f}=\frac{\rho g}{3 \mu} f^{2} \frac{d h}{d x}
$$

where $f$ is the film thickness, $g$ is the acceleration due to gravity, and the $\rho$ and $\mu$ are the fluid density and viscosity, respectively.

Multiplication of $v_{f}$, by the film-occupied cross section area yields the specific volumetric flux associated with film flow, $Q_{f}$, as 


$$
Q_{f}=\left(1-S_{c}\right) A \frac{\rho g}{3 \mu} f^{3} \frac{d h}{d x}
$$

where $A$ is the specific perimeter per unit area of the porous media, $\left(1-S_{c}\right)$ is the effective factor and $\left(1-S_{c}\right) A f$ accounts for the cross section area where film flow occurs. When $S_{c}$ is equal to 1 , it means that there is no space for film water to move.

Then, the film conductivity $K_{f}$ is

$$
K_{f}=\left(1-S_{c}\right) A \frac{\rho g}{3 \mu} f^{3}
$$

By setting the film conductivity at $S_{r}\left(S_{c}=0\right)$ as $K_{f}^{r}$, equation (16) can be rewritten as

$$
K_{f}=\left(1-S_{c}\right) K_{f}^{r}\left(\frac{f}{f_{r}}\right)^{3}
$$

where $f_{r}$ is the film thickness at $S_{r}$.

When the film is relatively thick $(>10 \mathrm{~nm})$, the ionic-electrostatic force dominates, the scaled film thickness can be expressed as (Tokunaga, 2009; 2011)

$$
\frac{f}{f_{r}}=\left(\frac{4 \delta / \lambda+\rho g h}{4 \delta / \lambda+\rho g h_{r}}\right)^{-0.5}
$$

where $\delta$ is the surface tension, $\lambda$ is the grain diameter.

The matric suction, $h r$, can be regarded as the critical suction under which films control viscous flow (Corey and Brooks, 1999). During drainage, Haines (1930) showed that $\rho g h_{r} \approx 26 \delta / \lambda$ in a mono-disperse close packing of spherical grains. Thus, equation (18) can be rewritten as

$$
\frac{f}{f_{r}}=\left(\frac{2}{15}+\frac{13 h}{15 h_{r}}\right)^{-0.5}
$$

Substituting equation (19) into equation (17) yields 


$$
K_{f}=\left(1-S_{c}\right) K_{f}^{r}\left(\frac{2}{15}+\frac{13 h}{15 h_{r}}\right)^{\tau}
$$

where $\tau=-1.5$ for smooth sphere grains.

Equation (20) is for the drainage process. While in a process towards saturation, the critical matric suction is close to $9.1 \delta / \lambda$ (Haines, 1930), resulting in a slightly different form for the film conductivity. However, with $K_{f}^{r}$ being a free fitting parameter, equation (20) can be used for both drainage and wetting processes. The impact of the different constant in the brackets can be ignored because film flow is only important at high matric suctions where $h / h_{r}$ has very large values. Nevertheless, this constant is kept to make sure that $K_{f}$ has a finite value when $h$ approaches zero.

Tokunaga's (2009) film thickness model (equation 18) accounts for the condition of smooth sphere grains, and the ionic strength is assumed to be low $\left(\leq 10^{-1} \mathrm{~mol} \mathrm{~m}^{-3}\right)$. These assumptions may not hold for real porous media. Here, the medium properties, such as surface roughness and particle non-uniformity, are assumed to be all reflected in the freely fitted parameter, $K_{f}^{r}$, and $\tau$ is kept constant as -1.5 as in many other models (Lebeau and Konrad, 2010; Zhang, 2011; Peters, 2013). The validity of such assumptions is tested with different soils, as shown in the next section.

It should be noted that for a very thin film (less than $10 \mathrm{~nm}$ ), film flow is likely to be impacted by modified viscosity at the particle surface, that is, the viscosity $\mu$ is also a function of the distance to the solid surface (Tuller and Or, 2001). Additionally, the molecular force becomes important for the very-thin film (Tokunaga, 2011). A more complex conductivity function is required to represent water movement in the very-thin film (Tuller and Or, 2001; Lebeau and Konrad, 2010; Tokunaga, 2011). In 
this study, such a very-thin film is not included considering the hydraulic conductivity would be extremely low and its contribution to water flow is insignificant.

In cases that the hydraulic conductivity data are given with water content data, it is convenient to express the film conductivity as a function of saturation. By estimating the film thickness with water content, Wang et al. (2013) gave the film conductivity expression as a power function of film water content, written as

$$
K_{f}=\left(1-S_{c}\right) K_{f}^{r}\left(\frac{\theta_{f}}{\theta_{r}}\right)^{3}
$$

where $\theta_{f}$ is the water content associated with the film force. Equation (21) is in a simple form and isn't impacted by the surface and solution chemistry in unsaturated soils for it doesn't rely on the film thickness-matric suction relationship, (Wang et al., 2013). By using the new water retention curve (equation (4)), however, the water content cannot be distinguished into different components associated with the film and capillary forces, respectively. Approximation of all moisture below $\theta_{r}$ to be film water content, equation (21) results in a good agreement with observed conductivity data for soils with coarse textures, it overestimates the film conductivity for fine soils (not shown). This is because the water content retained in the very fine pores might account for a high possession in the total water content below $\theta_{r}$. Note that for the capillary conductivity model (equation (13)), the water content below $\theta_{r}$ is totally ascribed to film force. This assumption, although not necessarily realistic, has a little impact on the capillary conductivity, which becomes extremely small in comparison with the film conductivity in the range drier than $\theta_{r}$. 
Here an approximate method is adopted to express the film conductivity as a function of saturation. Campbell and Shiozawa (1992) illustrated that the water content below $\theta_{r}$ will decrease linearly with the log-scale matric suction. Then, the matric suction and film water content relationship can be expressed as

$$
\theta=\theta_{r}\left(1-\frac{\ln \left(h / h_{r}\right)}{\ln \left(h_{0} / h_{r}\right)}\right)
$$

Equation (22) is used to derive $h(\theta)$ for suctions higher than $h_{r}$ and equation (5) is used for suctions lower than $h_{r}$. Substituting the results into equation (20) yields

$$
K_{f}(S)= \begin{cases}\left(1-S_{c}\right) K_{f}^{r}\left[\frac{2}{15}+\frac{13\left(e^{S^{-1 / m}}-e\right)^{1 / n}}{15 \alpha h_{r}}\right]^{\tau} & \text { for } S \geq S_{r} \\ K_{f}^{r}\left[\frac{2}{15}+\frac{13}{15}\left(\frac{h_{0}}{h_{r}}\right)^{\left(1-S / S_{r}\right)}\right]^{\tau} & \text { for } S<S_{r}\end{cases}
$$

It should be noted that equation (23) is only recommended when the soil hydraulic conductivity data is provided with the water content data.

\subsubsection{The Total Hydraulic Conductivity Function}

The total hydraulic conductivity is defined as

$$
K=K_{c}+K_{f}
$$

where $K\left[\mathrm{~L} \mathrm{~T}^{-1}\right]$ is the total hydraulic conductivity for liquid flow. Substitution of equations (13) and (20) into equation (24) yields

$$
K= \begin{cases}K_{s} S_{c}^{l}\left[1-\left(\frac{1-S^{1 / m}}{1-S_{r}^{1 / m}}\right)^{1-1 / n}\right]^{2}+\left(1-S_{c}\right) K_{f}^{r}\left(\frac{2}{15}+\frac{13 h}{15 h_{r}}\right)^{\tau} & \text { for } S \geq S_{r} \\ K_{f}^{r}\left(\frac{2}{15}+\frac{13 h}{15 h_{r}}\right)^{\tau} & \text { for } S<S_{r}\end{cases}
$$

In summary, the proposed new model is an extension and modification of the FX 
model, accounting for both film and capillary forces. Thus it is referred as the EMFX model. In comparison with the model developed by Fredlund et al., (1994), the calculation of capillary conductivity is simplified in the EMFX model. The soil water retention curve is represented by equation (4), the total hydraulic conductivity function is described in equation (25). When the conductivity data are provided with water contents, the total conductivity function is represented by substituting equations (14) and (23) into equation (24).

\section{Model testing and demonstration}

\subsection{Data for model testing}

To test the model performance, the soil hydraulic data should cover very dry conditions. Only a small number of published studies meet this requirement. Of them sixteen data sets were chosen for the model testing, with soil texture ranging from

sand to clay (Table 1). Soils 1 to 12 are the same as those used in Lebeau and Konrad (2010), reported in different literature (Pachepsky et al., 1984; Mehta et al., 1994; Fujimaki and Inoue, 2003a, 2003b; Mualem, 1976b; Nemes et al., 2001). Four clay soils were selected from the UNSODA database (Schindler et al., 1985; Becher, 1970) to test the model performance with soils of finer textures.

(Table 1 near here)

\subsection{Parameter Optimization}


The objective function $\Phi(b)$ to be minimized during the parameter estimation process is defined as the same as in Peters (2013),

$$
\Phi(b)=w_{\theta} \sum_{i=1}^{n_{\theta}}\left[\theta_{i}-\hat{\theta}_{i}(b)\right]^{2}+w_{k} \sum_{i=1}^{n_{K}}\left[\log _{10}\left(K_{i}\right)-\log _{10}\left(\widehat{K}_{i}(b)\right)\right]^{2}
$$

where $n_{\theta}$ and $n_{K}$ are the number of data pairs for the retention and the conductivity function, $\theta_{i}$ and $K_{i}$ are the measured water content and hydraulic conductivity while $\hat{\theta}_{i}$ and $\widehat{K}_{i}$ are the model estimated values, respectively. We set $w \theta=10000$ and $w k$ $=16$ as the weights of water content and conductivity data following Peters (2013), $b$ $=\left(h_{r}, \alpha, n, m, l, K_{f}^{r}\right)$ is the parameter vector used for optimization. When $l$ is set as 0.5 as suggested by Mualem (1976a), only five parameters are to be optimized.

The root mean squared errors, $\mathrm{RMSE}_{\theta}$ and $\mathrm{RMSE} \log _{\mathrm{K} r}$, are introduced for evaluating the model performance, defined as

$$
R M S E_{\theta}=\sqrt{\frac{1}{n_{\theta}} \sum_{i=1}^{n_{\theta}}\left(\theta_{i}-\hat{\theta}_{i}\right)^{2}}
$$

and

$$
R M S E_{\log K r}=\sqrt{\frac{1}{n_{K}} \sum_{i=1}^{n_{K}}\left(\log _{10}\left(K_{i}\right)-\log _{10}\left(\widehat{K}_{i}\right)\right)^{2}}
$$

Equation (26) is minimized with the SCE-UA method proposed by Duan et al. (1992).

\section{Results and Discussion}

\subsection{Model Testing}

(Table 2 near here) 
Figures 2 to 8 illustrate the model testing results for the sixteen soil data. The proposed EMFX model describes these soil hydraulic data over the entire matric suction range, both for the water retention curve and the hydraulic conductivity function. The fitting parameters are summarized in Table 2. Note that the hydraulic conductivity data of Masa loamy sand, Adelanto loam, Pachapa loam and Shonai sand were provided with the water content, in these cases, the saturation based film conductivity function (equation (23)) was used in the model testing. The results are grouped depending on the soil textures.

\section{(Figure 2 near here)}

Figures 2 and 3 illustrate the capabilities of the EMFX model for Sandy loam, Gilat loam, Adelanto loam and Pachapa loam. The EMFX model fits the water retention data well for all loam soils, with the RMSE $\theta$ in a range from 0.005 to 0.008 . The relatively poor performance is resulted for Adelanto loam, which has the highest water content corresponding to the critical matric suction. For the conductivity data, the transition from the capillary zone to the film range is well reflected, the critical matric suction for the film-dominated flow regime is close to $10^{3} \mathrm{~cm}$ for all loams except for Gilat loam, which has a much smaller value, about $5 \times 10^{2} \mathrm{~cm}$. The corresponding critical water content ranges from 0.15 to 0.23 . The slope of " $-1.5 "$ $\log -\log$ scale relationship (i.e., $\tau=-1.5$ ) yields a very good agreement with the conductivity data under conditions of low water contents, particularly for Sandy loam 
and Pachapa loam. The relatively poor performance for Adelanto loam might be associated with the relatively poor agreement with the water retention data. For Gilat loam, there is a small underestimation of the hydraulic conductivity at very high suctions. Peters (2013) attributed this to the impact of vapor diffusion. This underestimation can also be avoided by setting $\tau$ as -1.0 (not shown), suggesting that the problem may be related to the physical mechanism represented in the log-scale slope.

(Figure 3 near here)

Figure 4 shows the fitting results for Poederlee loamy sand and Masa loamy sand. The fitted critical matric suction is close to $10^{4} \mathrm{~cm}$ for the two loamy sands. For Poederlee loamy sand, the fitted results show that only two conductivity data is in the film range. The EMFX model matches the conductivity data well for Masa loamy sand while it slightly overestimates the water content at the suction of $10^{5} \mathrm{~cm}$.

(Figure 4 near here)

Figures 5 and 6 present the fitting results for five sand soils, including Rehovot sand, Berlin medium sand 4, Poederlee sand, Hupsel sand and Shonai sand. For these sand soils, the critical matric suctions have a much smaller value, close to $10^{2} \mathrm{~cm}$. 


\section{(Figure 5 near here)}

Although the EMFX model exhibits the transition from the capillary zone to the film range, it yields somewhat poor results for sand soils, particularly in terms of conductivity data. The RMSE $\theta$ and $\mathrm{RMSE}_{\log K r}$ range from 0.004 to 0.015 and 0.184 to 0.347, respectively. For the water retention data, the EMFX model slightly underestimates the water content for Poederlee sand and Shonai sand at high matric suctions. For the conductivity data, a fixed log-scale slope of "-1.5" (i.e., $\tau$ in equation 20) doesn't match well with the measured data at the film-dominated range. The EMFX model overestimates the conductivity when the matric suction is higher than $10^{2} \mathrm{~cm}$. The molecular forces might be important for film thickness less than 10 $\mathrm{nm}$, corresponding to a suction of about $10^{4} \mathrm{~cm}$ (Lebeau and Konrad, 2010). Additionally, a dominant molecular force in the film range would yield a log-scale slope of "-1.0", which deviates more significantly from the observed conductivity data (shown in next section). Thus, the overestimation cannot be simply explained by the impact of the modified viscosity and the molecular forces. This overestimation of sand soils can also be found in figure 9 of Peters (2013). This may indicate that the assumption of a fixed $-1.5 \log$-scale slope, derived for smooth sphere grains, may not work well for sand soils. Detailed discussion is given in the next section.

(Figure 6 near here) 
Figures 7 and 8 show the model testing results for soils with much finer textures, including one sandy clay and four clay soils. The EMFX model fits the experiment data very well for Pachapa fine sandy clay, with $\mathrm{RMSE}_{\theta}$ of 0.008 and $\mathrm{RMSE}_{\log K r}$ of 0.118 , respectively. The fitting result is in close agreement with the experimental data for Seelow clay 1, Seelow clay 2 and Hollern clay 1. However, for Hollern clay 2, the conductivity model shows a significant difference from the measured data in the dry range. The slope of the log-scale conductivity data yields a large value than -1.5 . Actually, setting the slope $\tau$ as -1 would significantly improve the model performance. The critical matric suction for four clays is close to $10^{3} \mathrm{~cm}$, corresponding to very high water contents. This result indicates that the film flow can be dominant in a wide range of water contents for clay soils.

(Figure 7 near here)

Note that the water retention data for Seelow clay 1 and Hollern clay 1 yield bimodal properties, which has been well captured by the EMFX model.

\section{(Figure 8 near here)}

For Masa loamy sand, Adelanto loam, Pachapa loam and Shonai sand, the saturation based film conductivity function (equation (23)) was used in the model testing. The proposed EMFX model describes the conductivity function well for all 
the four soils. Actually, the saturation based film conductivity function (equation (23)) produces comparable results with the matric suction based model (equation (20)) (not shown).

The main drawback of the EMFX model is that the proposed hydraulic conductivity function associated with the capillary force is based on an approximate method, which, in its physics meaning, might not be true for all $n$ and $m$ values. Nevertheless, the model testing results with sixteen different data sets suggest that the approximate conductivity function has enough flexibility in describing the capillary conductivity of different soils.

\subsection{The Film Conductivity Model}

The film conductivity model used here is developed for a porous media of smooth sphere grains, with the assumption that the surface roughness and particle non-uniformity are all reflected in the free fitting parameter, $K_{f}^{r}$. For simplicity, the $\log$-scale slope $\tau$ is kept constant as -1.5 , as in other models (Lebeau and Konrad, 2010; Zhang, 2011; Peters, 2013).

\section{(Figure 9 near here)}

Based on the fitted $h_{r}$, the scaled film conductivity and matric suction relationship are presented in figure 9. The soils are grouped into three types, loam, sand and clay. For all soils, the slope has a value of -1.28 with $R^{2}$ equals 0.80 (not shown in figure 9). However, the slopes for individual soil types vary from -2.30 to -1.18 . The slope 
of loam soils is -1.29 with an $R^{2}$ of 0.90 . The slopes of different loams vary from -1.50 (Sandy loam) to -1.12 (Gilat loam). The slopes of clay soils vary from -1.39 (Seelow clay 1 ) to -0.80 (Hollern clay 2 ), with a mean value of -1.18 .

While for sand soils, the slopes have more negative values, with the average value of -2.3 , and the difference between soils is also big, in a range from -4.20 (Hupsel sand) to -1.71 (Rehovot sand). This more negative log-scale slope cannot be explained by the impact of molecular forces as previously discussed. It might be associated with the large particle non-uniformity of sand soils. A prescribed -1.5 slope leads to the poor model performance for sand soils (figures 6 and 7).

\section{(Figure 10 near here)}

Figure 10 illustrates the model testing results for Poederlee sand and Berlin medium sand 4 with the log-scale slope prescribed as -2.0 . Clearly, the model yields much better results in comparison with that using a log-scale slope of -1.5 . Similar improvement can be achieved for all sand soils.

According to Tokunage $(2009 ; 2011)$, the log-scale slope of -1.5 means the ionic-electrical force controls flow in film while the log-scale slope would be -1.0 for the molecular-force dominated film flow regime. It is interesting to see that the low and upper bound of loam and clay soils happen to be close to -1.5 and -1.0 , respectively. However, it is difficult to tell whether this variation of slopes is associated with the different dominant forces in controlling film flow or with the 
media properties such as surface roughness.

In summary, for the film hydraulic model, a constant log-scale slope of -1.5 might be reasonable for soils with a fine texture. For sand soils, it is suggested to vary this slope to have a more negative value (e.g., -2) to improve the model performance.

\subsection{Comparison with the PDI Model}

(Table 3 near here)

The PDI model (see Appendix B) developed by Peters (2013) and improved by Iden and Duner (2014) was introduced for comparison with the EMFX model. The fitted $\Phi_{\min }, \mathrm{RMSE}_{\theta}$ and RMSE $\log _{\mathrm{K} r}$ values of the PDI model were given in Table 3. The $\Phi_{\min }$ values of the PDI model were fitted using the optimization procedure proposed by Duan et al., (1992). Note that we didn't use the smooth strategy for SWRC of the PDI model since it doesn't improve the result of optimization. The small difference between the fitted $\Phi_{\min }$ values here and those of Iden and Duner (2014) might be attributed to the different optimization procedure.

Table 3 illustrates that for most soils, the EMFX model yields better agreement with the measured data, both for the water retention and conductivity data. The $\mathrm{RMSE}_{\log K r}$ of the EMFX model is in a range of 0.046 to 0.347 , with a mean value of 0.196 and the RMSE $\theta$ ranges from 0.003 to 0.015 , with a mean value of 0.008 . The PDI model shows a much higher $\mathrm{RMSE}_{\log K r}$ and $\mathrm{RMSE}_{\theta}$, in the range from 0.082 to 0.442 and from 0.006 to 0.027 , with the mean value of 0.287 and 0.015 , respectively. 
Note that when $l$ is set as 0.5 , the EMFX model also yields good performance, with the fitted $\Phi_{\min }$ close to those treat $l$ as a free fitting parameter (Table 3 ).

In comparison with the PDI model, the EMFX model presents slightly poor results for Pachapa fine sandy clay, Poederlee sand and Poederlee loamy sand, but the difference is insignificant. For example, with the EMFX model and the PDI model, the $\Phi_{\min }$ values for Poederlee loamy sand are 18.3 and 18.1, respectively. The Adelanto loam is the only data set for which the PDI model yields a much better result. The $\Phi_{\min }$ values of the EMFX model and the PDI model are 19.3 and 8.5, respectively. For other twelve soils, the EMFX model shows much better performance, both for the water retention and conductivity data. Figure 11 illustrates the performance of the EMFX model and the PDI model for three data sets, including one sand, one loam and one clay.

\section{(Figure 11 near here)}

From table 3 and figure 11, it is clear that the water retention data is better fit by the EMFX model. The transition of water content from the capillary dominated zone to the film dominated zone is also better represented by the EMFX model. The PDI model describes the total water content as a sum of the water associated with the capillary force and film force, respectively. The water retention function for film flow is simply represented by a log-scale liner equation (equation (B4) in Appendix B), which shows less flexibility in comparison to the EMFX water retention function. 
This less flexibility can explain the relatively poor performance of the PDI model for loam and sand soils. However, this is not the case for clay soils, where the PDI model also presents poor agreement at high saturation where the capillary force dominates (figure 11). This mismatch at high saturation can be attributed to the simplified scaling method for capillary-associated SWRC in the PDI model (equation (B2) in Appendix B). Peters (2013) used the VG model to describe the water retention data associated with the capillary force. As an improvement, Iden and Duner (2014) introduced a scaling method (equation (B2)) to make sure the capillary force-associated water content decreases to zero at a suction of $h_{0}$. For clay soils with much finer textures, the VG model will result in a much higher saturation at the suction of $h o$, thus, this oversimplified scaling method would significantly influence the calculated retention at high saturations, yield poor performance to fit the water retention data. In addition, the PDI model cannot capture the bimodal structure of the water retention data presented in Seelow clay 1 and Holly clay 1.

The EMFX conductivity model is associated with SWRC, it also shows better agreement with the experimental data, indicating that the approximately closed-form equation for the capillary conductivity provides a good description of the hydraulic conductivity data for different soils.

\subsection{Modelling Soil Hydraulic Properties under Dry Conditions}

To extend the commonly used soil hydraulic models to dry conditions is crucial in understanding and describing the evaporation process, root water uptake and many other processes in water-limited environments. Recently, including the film flow into 
soil hydraulic models has been recognized as a big progress in hydraulic model development (Durner et al., 2014). However, problems, such as the mathematical discontinuity in the SWRC representation, and the accuracy of the film model in describing different soils, are yet to be addressed. The EMFX model has addressed the mathematical discontinuity problem. It is also found that the commonly used constant "-1.5" log-scale slope for film flow in the previous studies (Lebeau and Konrad, 2010; Zhang, 2011; Peters, 2013) might not be appropriate for all soils, particularly for sand soils. Based on the model fitting results, the critical matric suction for film flow to be dominating is in the range of $10^{2}$ to $10^{3} \mathrm{~cm}$, depending on the soil texture. This critical matric suction is common under field conditions. This calls for more attention to and an increasing need of investing film flow in unsaturated soils and incorporating film flow in variably saturated flow and transport modeling.

\section{Conclusions}

A new soil hydraulic model has been developed as an extension and modification of Fredlund and Xing (1994) model. The EMFX model describes the soil water-suction relationship and the hydraulic conductivity properties over the entire range of matric suctions. The new soil water retention curve is mathematically continuous at the whole moisture range and the new conductivity function has a simple form. In addition, the new model has capacity to capture the bimodal properties presented in structured and aggregated soils. The EMFX model has only one more parameter than commonly used soil hydraulic models which only account for capillary force. 
Model testing with sixteen different published data shows good performance for the water retention curve and the conductivity function in a range from saturation to very dry conditions. While the new model describes the soil hydraulic properties of the soils with fine textures well, it slightly overestimates the hydraulic conductivity of sand soils at very high suctions, indicating the constant log-scale slope of -1.5 doesn't hold for all soils. A slope of -2.0 might be more appropriate for sand soils.

The new model introduces one more free fitting parameter, $K_{f}^{r}$, to describe the film conductivity. Since the water retention data is easier to obtain than conductivity data, the future effort is recommended to predict $K_{f}^{r}$ from the SWRC.

\section{Appendix A}

The capillary conductivity of the EMFX model, $K_{c}$, is described as

$$
K_{c}=K_{s} S_{c}^{l}\left[\frac{\int_{S_{r} / / m}^{S^{1 / m}}\left(e^{1 / y}-e\right)^{-1 / n} y^{m-1} d y}{\int_{S_{r}^{1 / m}}^{1}\left(e^{1 / y}-e\right)^{-1 / n} y^{m-1} d y}\right]^{2}
$$

where $y=S^{1 / m}$.

Setting the integrated part as $\mathrm{F}(\mathrm{y})$,

$$
F(y)=\left[\frac{f(y)}{f(1)}\right]^{2}=\left[\frac{\int_{S_{r}^{1 / m}}^{S^{1 / m}}\left(e^{1 / y}-e\right)^{-1 / n} y^{m-1} d y}{\int_{S_{r}^{1 / m}}^{1}\left(e^{1 / y}-e\right)^{-1 / n} y^{m-1} d y}\right]^{2}
$$

The integrated part of the VG model with $\mathrm{m}=1-1 / \mathrm{n}$ is in the form of

$$
F_{V G}(y)=\left[\frac{\int_{S_{r}^{1 / m}}^{S^{1 / m}}(1-y)^{-1 / n} d y}{\int_{S_{r}^{1 / m}}^{1}(1-y)^{-1 / n} d y}\right]^{2}
$$


Note that the new model is in a similar form with VG model. Here, we assume that the difference between $F(y)$ and $F_{V G}(y)$ can be complemented by a power function of $S_{c}$, that is

$$
F(y)=S_{c}^{l_{1}} F_{V G}(y)
$$

where $l_{l}$ is a free fitted parameter.

\section{[Figure A1 near here]}

Figure (A1) indicates that with a proper $l_{l}$, the integrand part of the new model $F(y)$ can be approximately expressed in the form of equation (A4), particularly when $m$ has a small value. In the VG model, $m$ is assumed to be less than 1 , here the maximum value of $m$ is set as 1.5 . Note that when the capillary saturation approaches zero, the difference between these two integrand function becomes large, however, during this range, the capillary conductivity becomes very small in comparison with the film conductivity. Thus, we may approximately use equation (A4) to represent $F(y)$. Substitution of equation (A4) into (A1) yields,

$$
K_{c}=K_{s} S_{c}^{l}\left[\frac{\int_{S_{r}}^{S^{1 / m}}(1-y)^{-1 / n} d y}{\int_{S_{r}^{1 / m}}^{1}(1-y)^{-1 / n} d y}\right]^{2}=K_{s} S_{c}^{l}\left[1-\left(\frac{1-S^{1 / m}}{1-S_{r}^{1 / m}}\right)^{1-1 / n}\right]^{2}
$$

\section{Appendix B}

The PDI model is developed by Peters (2013) and improved by Iden and Duner (2014).

The total water content, $\theta$, is expressed as 


$$
\theta=\left(\theta_{s}-\theta_{r}\right) S_{c}+\theta_{r} S_{f}
$$

where $\theta_{s}$ is the saturation water content, $S_{c}$ is the capillary saturation. Iden and Duner (2014) introduced a scaling method so that $S_{c}$ decreases to zero at the suction of $h_{0}$, written as

$$
S_{c}(h)=\frac{\Pi(h)-\Pi\left(h_{0}\right)}{1-\Pi\left(h_{0}\right)}
$$

where $\Pi(h)$ is described using the VG model, as

$$
\Pi(h)=\left[1+(\alpha h)^{n}\right]^{-m}
$$

The film saturation $S_{f}=\theta_{f} / \theta_{r}$ is given by

$$
S_{f}= \begin{cases}X_{m}\left(1-\frac{\ln \left(1+h / h_{a}\right)}{\ln \left(1+h_{0} / h_{a}\right)}\right) & \text { for } h \geq h_{a} \\ 1 & \text { for } h<h_{a}\end{cases}
$$

where $h_{a}=1 / \alpha$ is the matric suction below which $S_{f}=1$, and

$$
X_{m}=\left(1-\frac{\ln (2)}{\ln \left(1+h_{0} / h_{a}\right)}\right)^{-1}
$$

For the conductivity model, the total conductivity, $K$, is written as

$$
K=K_{c}+K_{f}
$$

where the capillary conductivity, $K_{c}$, is given by

$$
K_{c}=(1-w) K_{s} S_{c}{ }^{l}\left[1-\left(\frac{1-\Pi^{1 / m}}{1-\Pi_{0}^{1 / m}}\right)^{m}\right]^{2}
$$

where $\Pi_{0}$ is the capillary saturation at the suction of $h_{0}$.

And, the film-associated conductivity is expressed as 


$$
K_{f}=w K_{s}\left(\frac{h_{0}}{h_{a}}\right)^{-1.5\left(1-S_{f}\right)}
$$

where $w$ is the weighting factor.

\section{Notation}

$A$ The specific perimeter per unit area of the porous media $\left(\mathrm{L} \mathrm{L}^{-2}\right)$.

$b$ The parameter vector used for optimization.

$\alpha$ Fitted parameter $\left(\mathrm{L}^{-1}\right)$.

$\beta$ Capillary model parameter.

$C(h)$ Correction function in the Fredlund and Xing (1994) model.

$c$ Scaling factor $(=0.01)$.

$d h / d x$ The matric suction gradient.

$e$ is Euler's number (the $e$ constant).

$f$ Film thickness (L).

$f_{r}$ Film thickness at $S_{r}(\mathrm{~L})$.

$f(S), f(1)$ and $f(y)$ The integrated functions of the capillary model.

$F(y)$ The integrated part of the capillary model in the EMFX model.

$F_{V G}(y)$ The integrated part of the capillary model in the VG model.

$\Gamma(h)$ Correction function in the EMFX model.

$\Phi(b)$ Objective function for optimization.

$g$ The acceleration due to gravity $\left(\mathrm{L} \mathrm{T}^{-2}\right)$

$\theta$ Volumetric water content $\left(\mathrm{L}^{3} \mathrm{~L}^{-3}\right)$.

$\theta_{r}$ Residual water content $\left(\mathrm{L}^{3} \mathrm{~L}^{-3}\right)$. 
$\theta_{f}$ Water content associated with the film force $\left(\mathrm{L}^{3} \mathrm{~L}^{-3}\right)$.

$\theta_{s}$ Saturated water content $\left(\mathrm{L}^{3} \mathrm{~L}^{-3}\right)$.

$\hat{\theta}_{i}$ Model estimated water content $\left(\mathrm{L}^{3} \mathrm{~L}^{-3}\right)$.

$h$ Matric suction (L).

$h_{r}$ Matric suction corresponding to the residual water content (L).

$h_{0}$ Matric suction where water content is zero (L).

$h_{\mathrm{a}}$ The critical matric suction in the PDI model, equals $1 / \alpha(\mathrm{L})$.

$h(S)$ Matric suction as a function of $S$.

$k$ Capillary model parameter.

$K$ The total hydraulic conductivity $\left(\mathrm{L} \mathrm{T}^{-1}\right)$.

$K_{c}$ Capillary conductivity $\left(\mathrm{L} \mathrm{T}^{-1}\right)$.

$K_{f}$ Film conductivity $\left(\mathrm{L} \mathrm{T}^{-1}\right)$.

$K_{f}(S)$ Film conductivity as a function of saturation $\left(\mathrm{L} \mathrm{T}^{-1}\right)$.

$K_{f}^{r} \quad$ Film conductivity at $S_{r}\left(\mathrm{~L} \mathrm{~T}^{-1}\right)$.

$\widehat{K}_{i}$ Model estimated conductivity values $\left(\mathrm{L} \mathrm{T}^{-1}\right)$.

$K_{s}$ Saturated hydraulic conductivity $\left(\mathrm{L} \mathrm{T}^{-1}\right)$.

$l$ Capillary model parameter.

$l_{l}$ Fitted parameter.

$\lambda$ Grain diameter $(\mathrm{L})$.

$m$ Fitted parameter.

$n$ Fitted parameter.

$n_{K}$ The number of conductivity data used for optimization. 
$n_{\theta}$ The number of retention data used for optimization.

$\mu$ Viscosity of the fluid $\left(\mathrm{M} \mathrm{L}^{-1} \mathrm{~T}^{-1}\right)$.

$\Pi(h)$ The unscaled capillary saturation in the VG model.

$\Pi_{0}$ The capillary saturation at the suction of $h_{0}$ in the VG model.

$Q_{c}$ The specific volumetric flux associated with the capillary force $\left(\mathrm{L} \mathrm{T}^{-1}\right)$.

$Q_{f}$ The specific volumetric flux associated with the film force $\left(\mathrm{L} \mathrm{T}^{-1}\right)$.

$\rho$ Density of the fluid $\left(\mathrm{M} \mathrm{L}^{-3}\right)$.

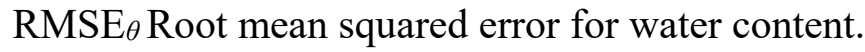

$\mathrm{RMSE}_{\log K r}$ Root mean squared error for log-scale conductivity.

$S$ Saturation degree.

$S_{c}$ The effective saturation where the capillary force dominates.

$S_{r}$ The saturation degree at the residual water content.

$S_{f}$ The film saturation degree.

$\tau$ Exponent in the film conductivity model.

$v_{f}$ The film thickness averaged film velocity $\left(\mathrm{L} \mathrm{T}^{-1}\right)$.

$w$ Weight factor for the conductivity in the PDI model.

${ }_{w} \theta$ Weight for the water content.

$w_{k}$ Weight for the conductivity data.

$X_{m}$ Scaling factor for the film water retention curve in the PDI model

$y$ Variable used for deriving the capillary conductivity, equals $S^{1 / m}$.

$\delta$ Surface tension $\left(\mathrm{N} \mathrm{L}^{-1}\right)$.

$S W R C$ Soil water retention curve. 
FX model The model developed by Fredlund and Xing (1994).

EMFX model The proposed model as an extension and modification of the FX model.

PDI model The model developed by Peters (2013) and improved by Iden and Duner (2014).

$V G$ model The model developed by van Genucthen (1980).

\section{Acknowledgments}

The research was supported by the Fundamental Research Funds for Central Universities, China University of Geosciences (Wuhan) (No. G1323521674) and by the National Science Foundation of China (Nos. 41272039 and U1403282). The first author wishes to thank China Scholarship Council for supporting his visiting research at Flinders University of South Australia. We wish to thank Marc Lebeau (Laval University, Quebec City, Canada), who kindly provided the data for soils 1 to 12 . Finally, the authors thank all anonymous reviewers for their very insightful and constructive comments on this and an early version of this manuscript.

\section{References}

Alexander, L., and R. W. Skaggs (1986), Predicting unsaturated hydraulic conductivity from the soil water characteristic, Trans. Am. Soc. Agric. Eng., 29(1), pp. 0176 - 0184, doi: 10.13031/2013.30123. 
Bear, J., Rubinstein, B., \& Fel, L. (2011). Capillary pressure curve for liquid menisci in a cubic assembly of spherical particles below irreducible saturation. Transport in porous media, 89(1), 63-73, doi: 10.1007/s11242-011-9752-7.

Becher, H. H. (1970). Eine Methode zur Messung der Wasserleitfähigkeit von Böden im ungesättigten Zustand. Technischen Universität, Fakultät für Gartenbau und Landeskultur.

Bird, R. B., W. E. Stewart, and E. N. Lightfoot (1960), Transport Phenomena, John Wiley, New York.

Brooks, R. H., and A. T. Corey (1964), Hydraulic properties of porous media, Hydrology Papers No. 3, pp. 1-27, Colorado State Univ., Fort Collins, CO, USA.

Burdine, N. T. (1953), Relative permeability calculations from pore size distribution data, Trans. Am. Inst. Min. Metall. Pet. Eng., 198, 71 - 78.

Campbell, G. S., and S. Shiozawa (1992), Prediction of hydraulic properties of soils using particle-size distribution and bulk density data, in Proceedings of the International Workshop on Indirect Methods for Estimating the Hydraulic Properties of Unsaturated Soil, edited by M. Th. van Genuchten, F. J. Leij, and L. J. Lund, pp. 317-328, Univ. of California, Riverside, CA, USA.

Corey, A. T., and R. H. Brooks (1999), The Brooks-Corey relationships, in Proceedings of the International Workshop on Characterization und Measurement of the Hydraulic Properties of Unsaturated Porous Media, edited by M. Th. van Genuchten, F. J. Leij, and L. Wu, pp. 13-18, Univ. of California, Riverside, CA, USA. 
Dexter, A. R., \& Richard, G. (2009). Tillage of soils in relation to their bi-modal pore size distributions. Soil and Tillage Research, 103(1), 113-118, doi:10.1016/j.still.2008.10.001.

Duan, Q., Sorooshian, S., \& Gupta, V. (1992), Effective and efficient global optimization for conceptual rainfall-runoff models. Water Resour. Res, 28(4), 1015-1031, doi: 10.1029/91WR02985.

Durner, W., Diamantopoulos, E., Iden, S. C., \& Scharnagl, B. (2014). Hydraulic properties and non-equilibrium water flow in soils. In Application of Soil Physics in Environmental Analyses (pp. 403-434). Springer International Publishing.

Fujimaki, H., and M. Inoue (2003a), A transient evaporation method for determining soil hydraulic properties at low pressure, Vadose Zone J., 2(3), 400 - 408, doi:10.2113/2.3.400.

Fujimaki, H., and M. Inoue (2003b), A flux - controlled steady - state evaporation method for determining unsaturated hydraulic conductivity at low matric pressure head values, Soil Sci., 168(6), $385 \quad-\quad 395, \quad$ doi:10.1097/ 00010694-200306000-00001.

Fredlund, D. G., and A. Q. Xing (1994), Equations for the soil-water characteristic curve, Can. Geotech. J., 31(4), 521 - 532, doi: 10.1139/t94-061.

Fredlund, D. G., Xing, A., \& Huang, S. (1994), Predicting the permeability function for unsaturated soils using the soil-water characteristic curve. Can. Geotech. $J .$, 31(4), 533-546, doi: 10.1139/t94-062 
Haines, W. B. (1930), Studies in the physical properties of soil. V. The hysteresiseffect in capillary properties, and the modes of moisture distributionassociated therewith, J. Agric. Sci., 20, 97 - 116, doi:10.1017/S002185960008864X.

Hoffmann-Riem, H., M. T. van Genuchten, and H. Flühler (1999), A General model for the hydraulic conductivity of unsaturated soils, in Proceedings of the International Workshop on Characterization and Measurement of the Hydraulic Properties of Unsaturated Porous Media, edited by M. T. van Genuchten, F. J. Leij, and L.Wu, pp. 31 - 42, Univ. of California, Riverside, Calif.

Iden, S. C., and W. Durner (2014), Comment to "Simple consistent models for water retention and hydraulic conductivity in the complete moisture range" by A. Peters, Water Resour. Res., 50, doi:10.1002/2014WR015937

Lebeau, M., and J.-M. Konrad (2010), A new capillary and thin film flow model for predicting the hydraulic conductivity of unsaturated porous media, Water Resour. Res., 46, W12554, doi:10.1029/2010WR009092.

Lenormand, R. (1990), Liquids in porous media, J. Phys.: Condensed Matter, 2, SA79-SA88, doi:10.1088/0953-8984/2/S/008.

Li, Y., and N. C. Wardlaw (1986), Mechanisms of nonwetting phase trapping during imbibition at slow rates, J. Colloid Interface Sci., 109, 473-486, doi:10.1016/0021-9797(86)90325-5. 
Mehta, B. K., S. H. O. Shiozawa, and M. Nakano (1994), Hydraulic properties of a sandy soil at low water contents, Soil Sci., 157(4), 208 - 214, doi:10.1097/00010694-199404000-00002.

Millington, R., and J. P. Quirk (1961), Permeability of porous solids, Trans. Faraday Soc., 57(8), $1200-1207$.

Mualem, Y. (1976a), A new model for predicting the hydraulic conductivity of unsaturated porous media, Water Resour. Res., 12(3), 513-521, doi:10.1029/WR012i003p00513.

Mualem, Y. (1976b), A Catalogue of the Hydraulic Properties of Unsaturated Soils, 100 pp., Technion, Israel Inst. of Technol., Haifa, Israel.

Nemes, A., M. G. Schaap, F. J. Leij, and J. H. M. Wösten (2001), Description of the unsaturated soil hydraulic database UNSODA version 2.0, J. Hydrol., 251(3 - 4), 151 - 162, doi:10.1016/S0022-1694(01)00465-6.

Or, D., and M. Tuller (1999), Liquid retention and interfacial area in variably saturated porous media: Upscaling from single - pore to sample - scale model, Water Resour. Res., 35(12), 3591 - 3605, doi:10.1029/1999WR900262.

Orr, F. M., Scriven, L. E., \& Rivas, A. P. (1975). Pendular rings between solids: meniscus properties and capillary force. Journal of Fluid Mechanics,67(04), 723-742.

Pachepsky, Y. A., R. A. Shcherbakov, G. Varallyay, and K. Rajkai (1984), On obtaining soil hydraulic conductivity curves from water retention curves (in Russian), Pochvovedenie, 10, 60 - 72. 
Peters, A. (2013), Simple consistent models for water retention and hydraulic conductivity in the complete moisture range, Water Resour. Res., 49, 6765-6780, doi:10.1002/wrcr.20548.

Richards, L. A. (1931), Capillary conduction of liquids in porous mediums, J. Appl. Phys., 1(5), 318-333.

Romano, N., Nasta, P., Severino, G., \& Hopmans, J. W. (2011). Using bimodal lognormal functions to describe soil hydraulic properties. Soil Science Society of America Journal, 75(2), 468-480, doi:10.2136/sssaj2010.0084.

Rossi, C., and J. R. Nimmo (1994), Modeling of soil water retention from saturation to oven dryness, Water Resour. Res., 30, 701 - 708, doi:10.1029/93WR03238.

Schaap, M. G., and F. J. Leij (2000), Improved prediction of unsaturated hydraulic conductivity with the Mualem-van Genuchten model, Soil Sci. Soc. Am. J., 64, 843 - 851, doi:10.2136/sssaj2000.643843x.

Schindler, U., Bohne, K., \& Sauerbrey, R. (1985). Comparison of different measuring and calculating methods to quantify the hydraulic conductivity of unsaturated soil. Zeitschrift für Pflanzenernährung und Bodenkunde, 148(6), 607-617, doi: 10.1002/jpln.19851480603.

Schneider, M., and K.-U. Goss (2012), Prediction of the water sorption isotherm in air dry soils, Geoderma, 170, 64 - 69, doi:10.1016/j.geoderma.2011.10.008.

Silva, O., and J. Grifoll (2007), A soil-water retention function that includes the hyper-dry region through the BET adsorption isotherm, Water Resour. Res., 43, W11420, doi:10.1029/2006WR005325. 
Toledo, P. G., R. A. Novy, H. T. Davis, and L. E. Scriven (1990), Hydraulic conductivity of porous media at low water content, Soil Sci. Soc. Am. J., 54, 673679, doi:10.2136/sssaj1990.03615995005400030007x.

Tokunaga, T. K. (2009), Hydraulic properties of adsorbed water films in unsaturated porous media, Water Resour. Res., 45, W06415, doi:10.1029/2009WR007734.

Tokunaga, T. K. (2011), Physicochemical controls on adsorbed water film thickness in unsaturated geological media, Water Resour. Res., 47, W08514, doi:10.1029/2011WR010676.

Tuller, M., Or, D., \& Dudley, L. M. (1999). Adsorption and capillary condensation in porous media: Liquid retention and interfacial configurations in angular pores. Water Resources Research, 35(7), 1949-1964.

Tuller, M., and D. Or (2001), Hydraulic conductivity of variably saturated porous media: Film and corner flow in angular pore space, Water Resour. Res., 37(5), 1257-1276, doi:10.1029/2000WR900328.

van Genuchten, M. Th. (1980), A closed-form equation for predicting the hydraulic conductivity of unsaturated soils, Soil Sci. Soc. Am. J., 44, 892-898, doi:10.2136/sssaj1980.03615995004400050002x.

Wang, Y., J. Ma, Y. Zhang, M. Zhao, and W. M. Edmunds (2013), A new theoretical model accounting for film flow in unsaturated porous media, Water Resour. Res., 49, 5021-5028, doi:10.1002/wrcr.20390. 
Zhang, Z. F. (2011), Soil water retention and relative permeability for conditions from oven-dry to full saturation, Vadose Zone J., 10, 1299-1308, doi:10.2136/vzj2011.0019.

Table 1. Soil properties of the Testing Data

\begin{tabular}{ccccc}
\hline No & Data Set & Reference $^{\mathrm{a}}$ & $\theta_{s}$ & $K_{s}(\mathrm{~cm} / \mathrm{d})^{\mathrm{b}}$ \\
\hline 1 & Sandy loam & A & 0.43 & 8.00 \\
2 & Shonai sand & B & 0.43 & 941.76 \\
3 & Masa loamy sand & C & 0.34 & 50.98 \\
4 & Gilat loam & D & 0.44 & 17.28 \\
5 & Pachapa loam & D & 0.46 & 17.28 \\
6 & Adelanto loam & D & 0.43 & 3.89 \\
7 & Pachapa fine sany clay & D & 0.33 & 12.10 \\
8 & Rehovot sand & D & 0.40 & 1100.0 \\
9 & Berlin medium sand 4 & E & 0.39 & 692.06 \\
10 & Poederlee loamy sand & E & 0.42 & 241.06 \\
11 & Poederlee sand & E & 0.42 & 164.16 \\
12 & Hupsel sand & E & 0.36 & 705.02 \\
13 & Seelow clay 1 & F & 0.49 & 5.00 \\
14 & Seelow clay 2 & F & 0.557 & 2.00 \\
15 & Hollern clay 1 & G & 0.555 & 20.00 \\
16 & Hollern clay 2 & G & 0.578 & 10.00 \\
\hline
\end{tabular}

${ }^{\text {a A }}$, Pachepsky et al. (1984); B, Mehta et al. (1994); C, Fujimaki and Inoue (2003a, 2003b); D, Mualem (1976b); E, Nemes et al. (2001); F, Schindler et al., (1985); G, Becher, (1970). ${ }^{\text {b For }}$ soils 15 and 16, the saturated conductivity data are fitted using the EMFX model. 
Table 2. The fitted parameters for the new model $b=\left(h r, \alpha, n, m, l, K_{f}^{r}\right)$

\begin{tabular}{ccccccc}
\hline Data Set & $\alpha(1 / \mathrm{cm})$ & $n$ & $K_{f}^{r}(\mathrm{~cm} / \mathrm{d})$ & $h_{r}(\mathrm{~cm})$ & $m$ & $l$ \\
\hline Sandy loam & 0.009 & 1.43 & $1.02 \times 10^{-4}$ & 1573 & 1.14 & 0.30 \\
Gilat loam & 0.023 & 4.60 & $2.38 \times 10^{-3}$ & 235 & 0.51 & 1.21 \\
Rehovot sand & 0.046 & 3.42 & $3.71 \times 10^{-4}$ & 74 & 1.49 & 0.98 \\
Pachapa fine sandy clay & 0.013 & 3.67 & $3.20 \times 10^{-4}$ & 719 & 0.59 & 0.75 \\
Berlin medium sand 4 & 0.032 & 9.00 & $4.23 \times 10^{-3}$ & 64.0 & 0.80 & 0.30 \\
Poederlee sand & 0.024 & 1.51 & $2.51 \times 10^{-3}$ & 556 & 1.41 & 1.72 \\
Hupsel sand & 0.044 & 4.88 & $1.44 \times 10^{-3}$ & 77 & 0.99 & -0.56 \\
Poederlee loamy sand & 0.034 & 1.81 & $1.47 \times 10^{-5}$ & 14990 & 0.81 & 2.77 \\
Masa loamy sand & 0.017 & 1.76 & $6.10 \times 10^{-5}$ & 4335 & 0.88 & 2.27 \\
Adelanto loam & 0.004 & 2.53 & $2.36 \times 10^{-3}$ & 1107 & 0.46 & -0.37 \\
Pachapa loam & 0.008 & 2.50 & $4.12 \times 10^{-3}$ & 534 & 0.77 & 0.21 \\
Shonai sand & 0.050 & 8.68 & $6.32 \times 10^{-2}$ & 52 & 0.74 & 0.77 \\
Seelow clay 1 & 0.015 & 1.58 & $6.79 \times 10^{-2}$ & 300 & 0.15 & -0.89 \\
Seelow clay 2 & 0.001 & 1.16 & $1.39 \times 10^{-2}$ & 785 & 0.21 & -0.12 \\
Hollern clay 1 & 0.005 & 1.10 & $4.08 \times 10^{-4}$ & 626 & 0.30 & 4.54 \\
Hollern clay 2 & 0.003 & 1.11 & $9.96 \times 10^{-3}$ & 349 & 0.27 & 8.00 \\
\hline
\end{tabular}


Table 3. Estimated $\Phi_{\min }, \mathrm{RMSE}_{\log K r}$ and $\mathrm{RMSE}_{\theta}$ values using EMFX and PDI $\operatorname{models}^{\mathrm{a}}$

\begin{tabular}{|c|c|c|c|c|c|c|}
\hline \multirow{2}{*}{ Data Set } & \multicolumn{3}{|c|}{ EMFX model } & \multicolumn{3}{|c|}{ PDI model } \\
\hline & $\Phi_{\min }$ & $\mathrm{RMSE}_{\log K r}$ & $\operatorname{RMSE}_{\theta}$ & $\Phi_{\min }$ & $\mathrm{RMSE}_{\log K r}$ & $\operatorname{RMSE}_{\theta}$ \\
\hline Sandy loam & $26.2(26.6)$ & 0.216 & 0.008 & 53.2 & 0.314 & 0.012 \\
\hline Gilat loam & $11.7(12.8)$ & 0.129 & 0.005 & 17.3 & 0.188 & 0.06 \\
\hline Rehovot sand & $27.6(30.4)$ & 0.257 & 0.004 & 106.8 & 0.483 & 0.011 \\
\hline Pachapa fine sandy clay & $14.2(14.6)$ & 0.118 & 0.008 & 13.6 & 0.106 & 0.008 \\
\hline Berlin medium sand 4 & $8.7(8.9)$ & 0.233 & 0.005 & 11.2 & 0.245 & 0.007 \\
\hline Poederlee sand & $18.8(19.7)$ & 0.184 & 0.012 & 18.7 & 0.202 & 0.011 \\
\hline Hupsel sand & $28.3(47.2)$ & 0.311 & 0.008 & 38.6 & 0.335 & 0.010 \\
\hline Poederlee loamy sand & $18.3(25.9)$ & 0.186 & 0.012 & 18.1 & 0.185 & 0.012 \\
\hline Masa loamy sand & $17.0(39.4)$ & 0.163 & 0.006 & 65.8 & 0.333 & 0.010 \\
\hline Adelanto loam & $19.3(21.4)$ & 0.190 & 0.008 & 8.5 & 0.082 & 0.006 \\
\hline Pachapa loam & $14.2(16.3)$ & 0.085 & 0.008 & 48.1 & 0.282 & 0.012 \\
\hline Shonai sand & 234.0 (252.3) & 0.347 & 0.015 & 293.9 & 0.376 & 0.0179 \\
\hline Seelow clay 1 & $7.8(10.0)$ & 0.125 & 0.006 & 110.7 & 0.307 & 0.027 \\
\hline Seelow clay 2 & $1.8(6.6)$ & 0.046 & 0.003 & 49.8 & 0.357 & 0.014 \\
\hline Hollern clay 1 & $35.7(40.5)$ & 0.270 & 0.006 & 78.4 & 0.350 & 0.011 \\
\hline Hollern clay 2 & $46.9(62.8)$ & 0.281 & 0.008 & 99.6 & 0.442 & 0.010 \\
\hline
\end{tabular}

${ }^{a}$ The lowest $\Phi_{\min }$ is highlighted in bold; for EMFX model, six parameters are needed for optimization with $b=\left(h_{r}, \alpha, n, m, l, K_{f}^{r}\right)$, when $l$ is set as 0.5 , the number of parameters reduces to five, the fitted $\Phi_{\min }$ is listed in brackets; for PDI model, five parameters are estimated for optimization with $b=\left(\theta_{r}, \alpha, n, l, w\right)$. 
a) $h_{r}=2000 \mathrm{~cm}, m=0.2$

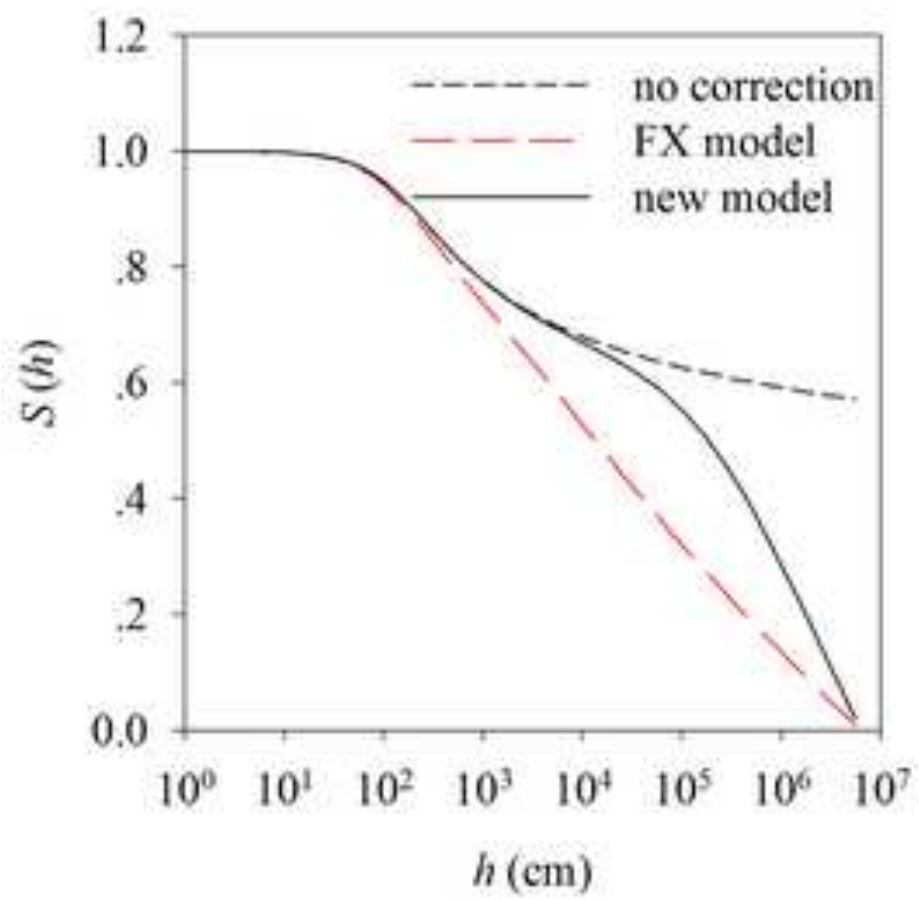

c) $h_{r}=200 \mathrm{~cm}, m=0.2$

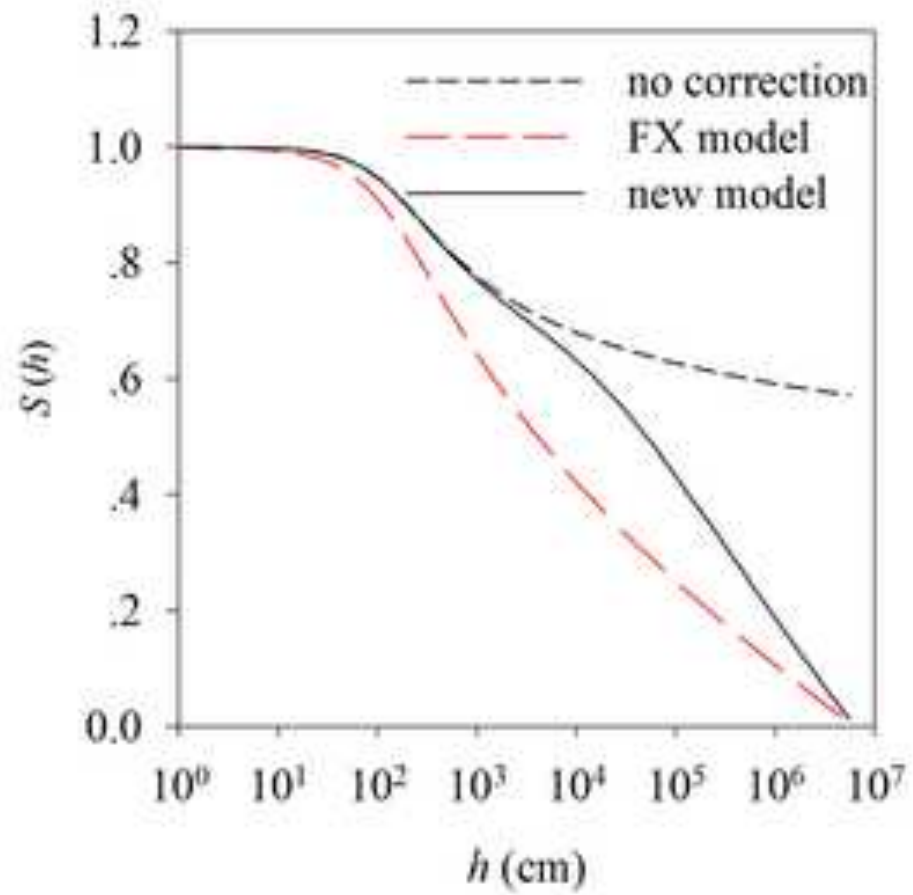

b) $h_{r}=2000 \mathrm{~cm}, m=0.8$

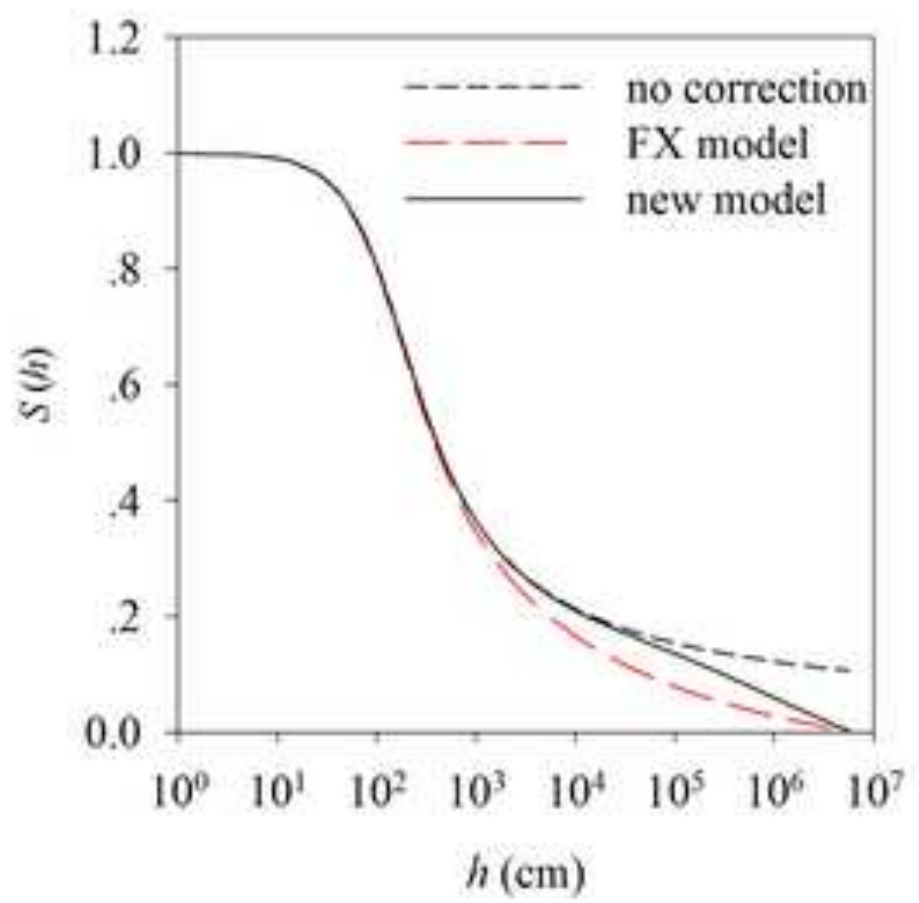

d) $h_{r}=200 \mathrm{~cm}, m=0.8$

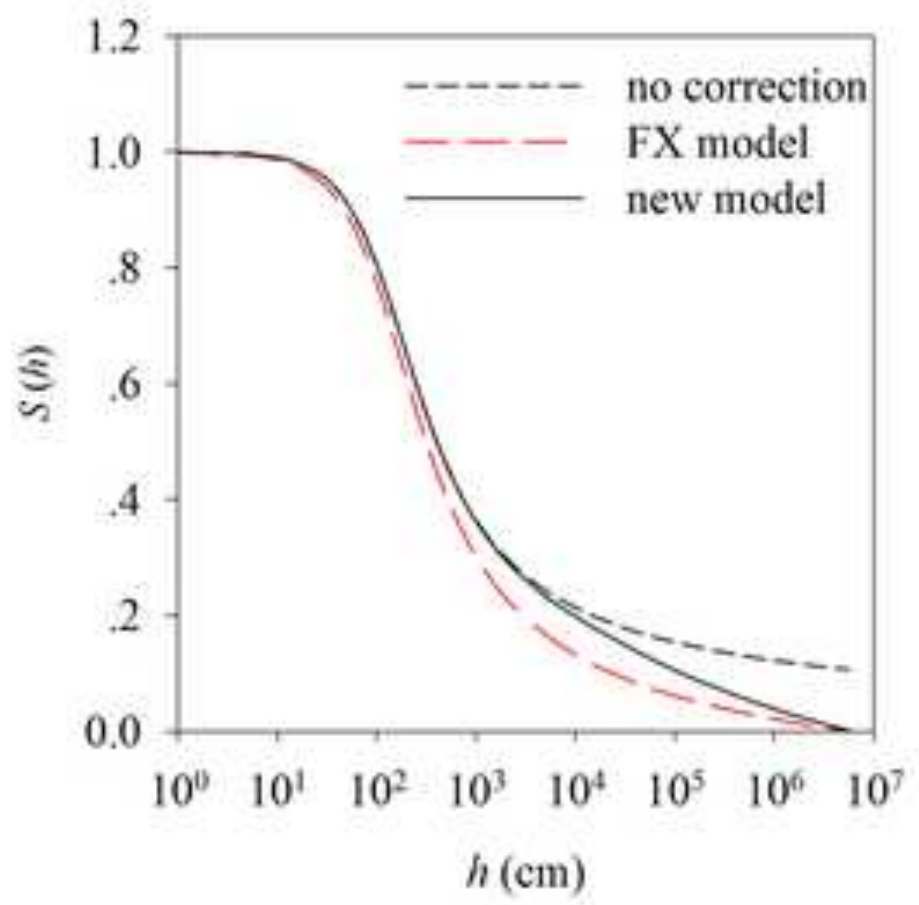


Sandy loam
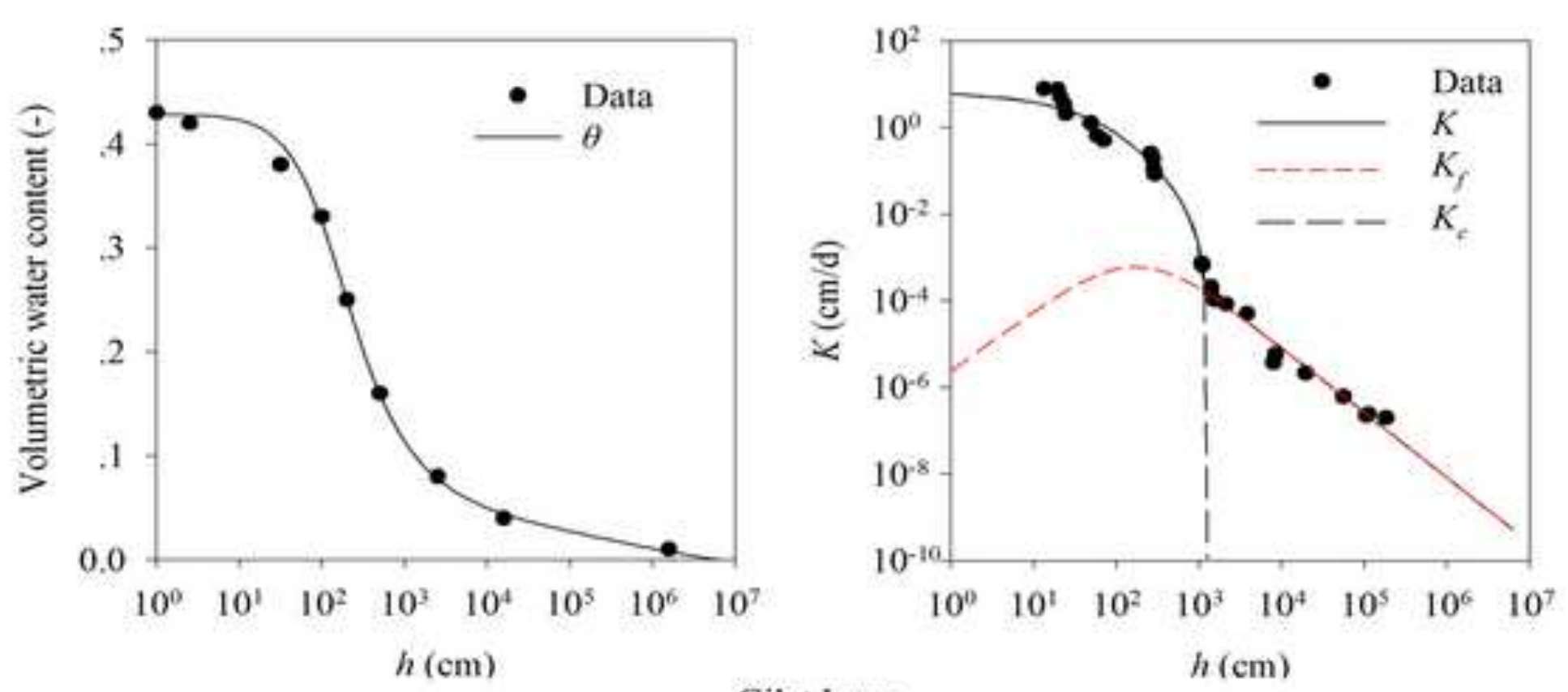

Gilat loam
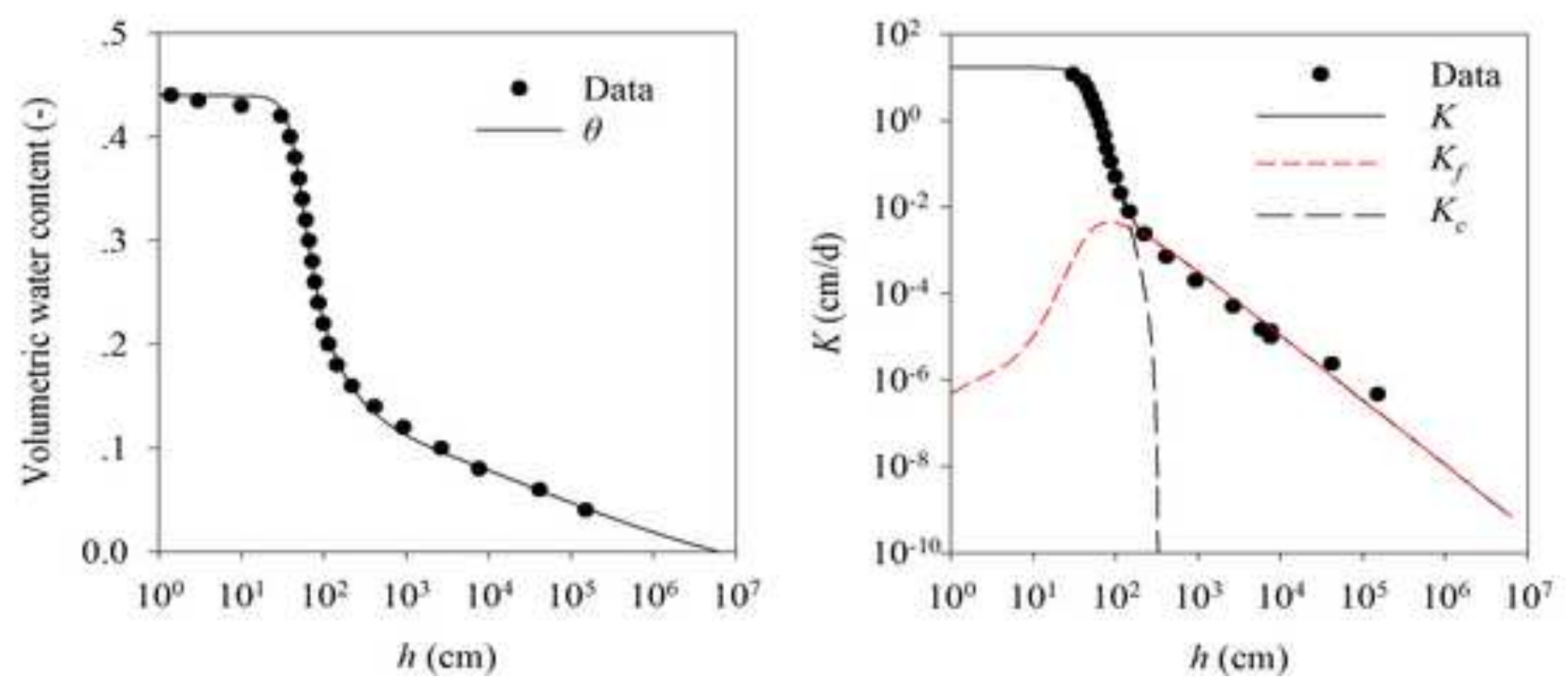
Adelanto loam
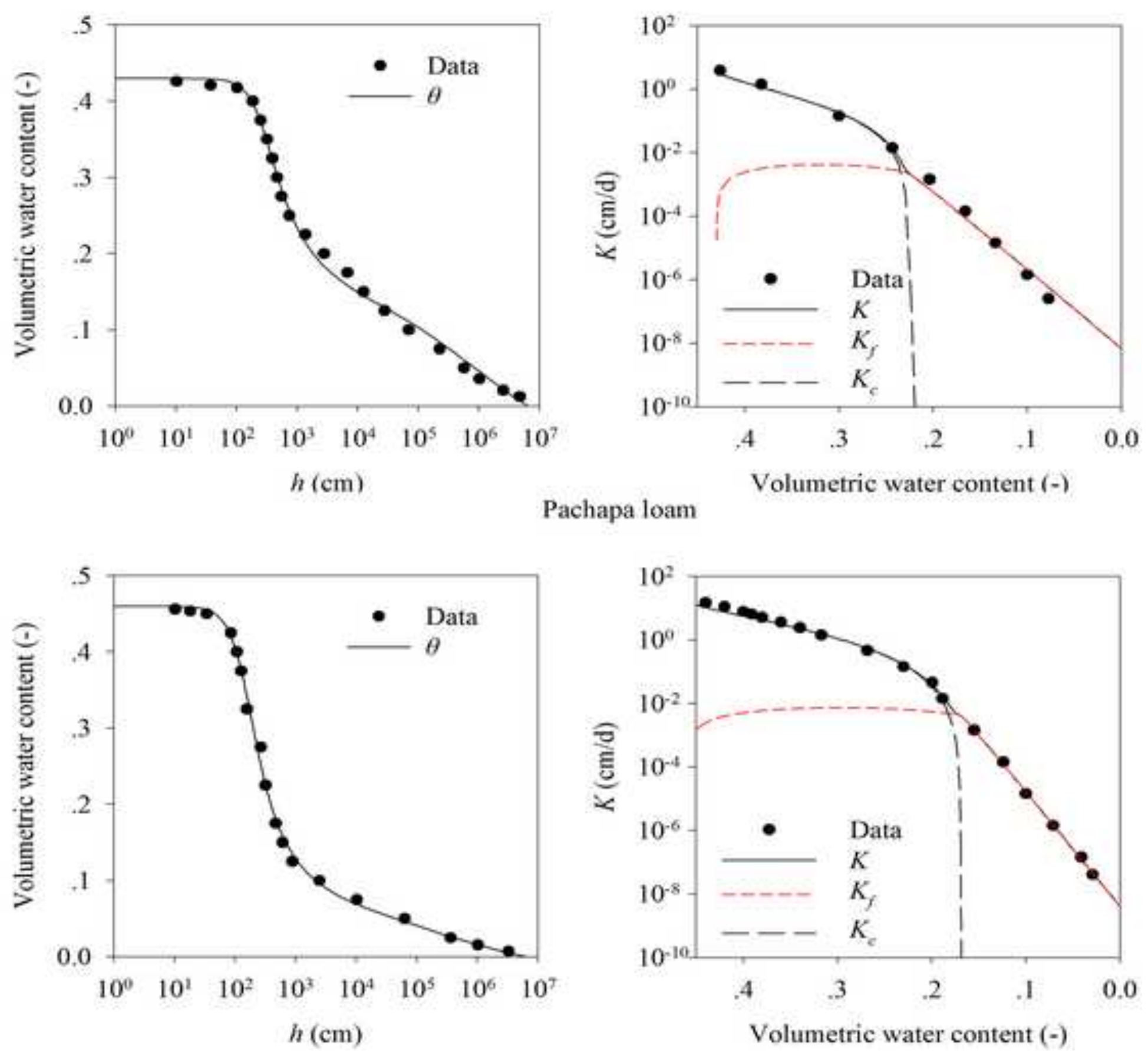
Poederlee loamy sand
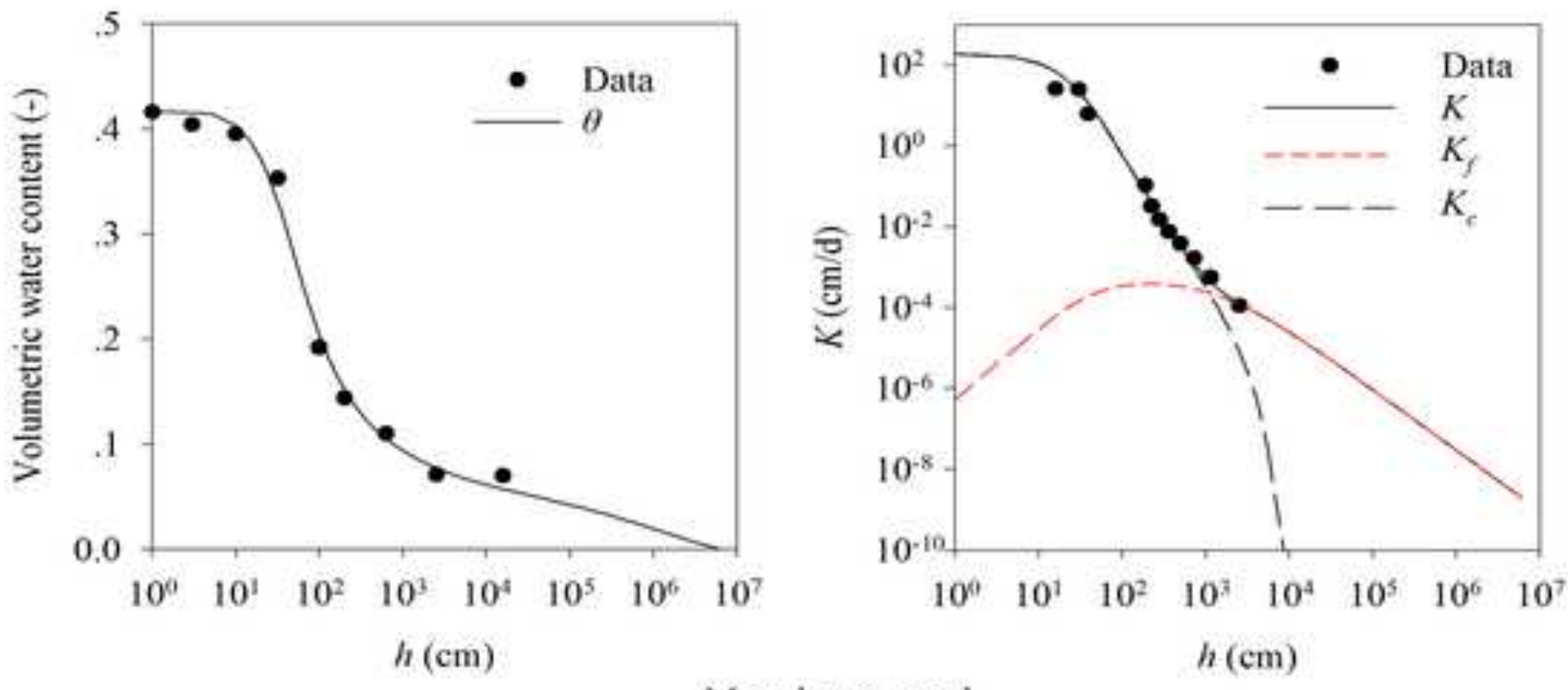

Masa loamy sand
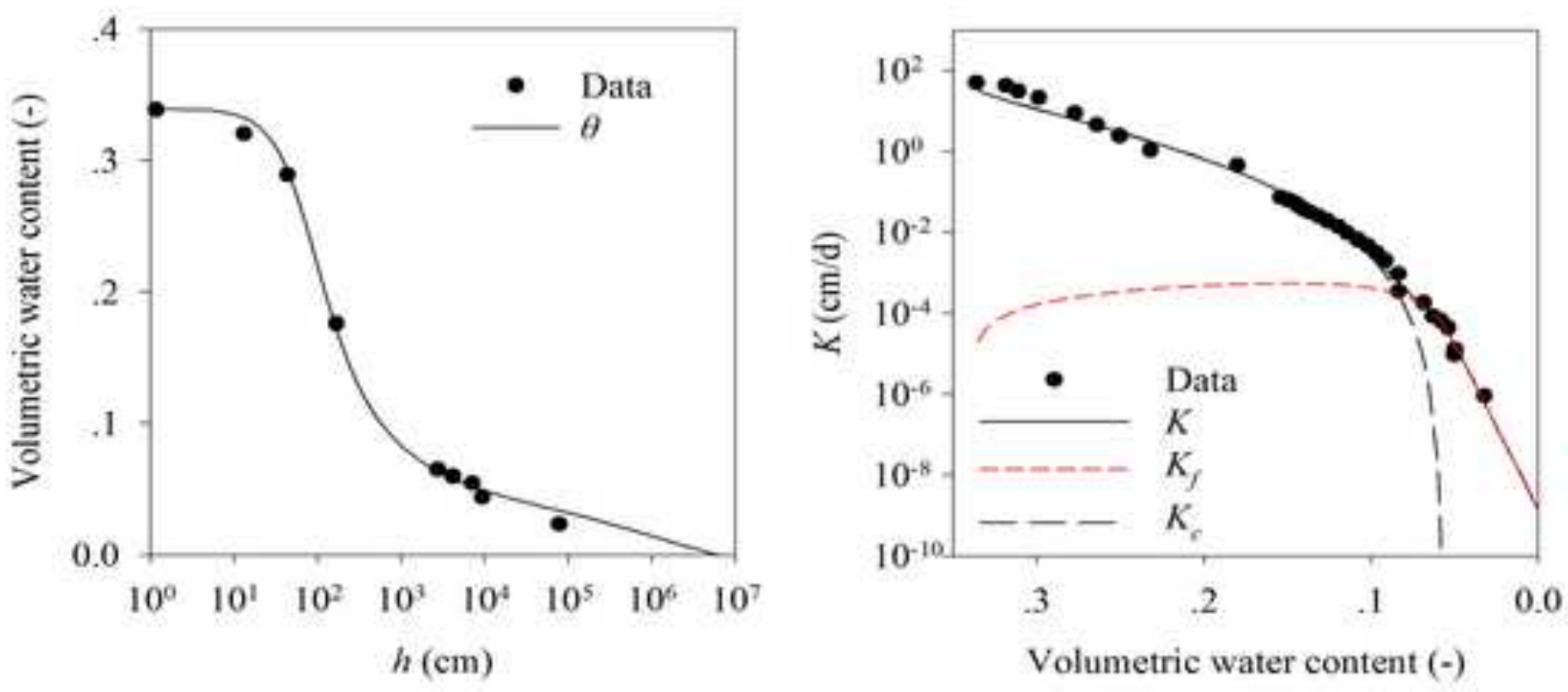
Figure 5

Rehovot sand
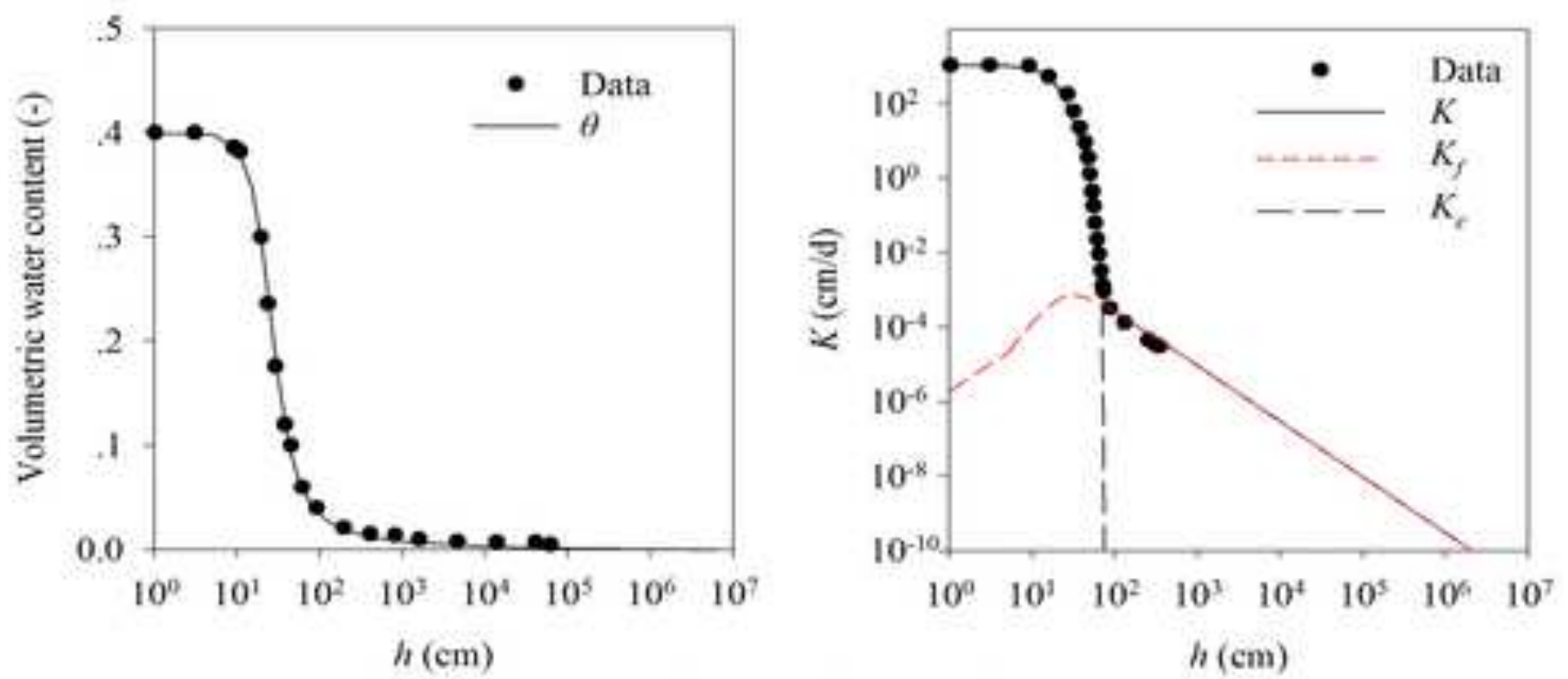

Berlin medium sand 4
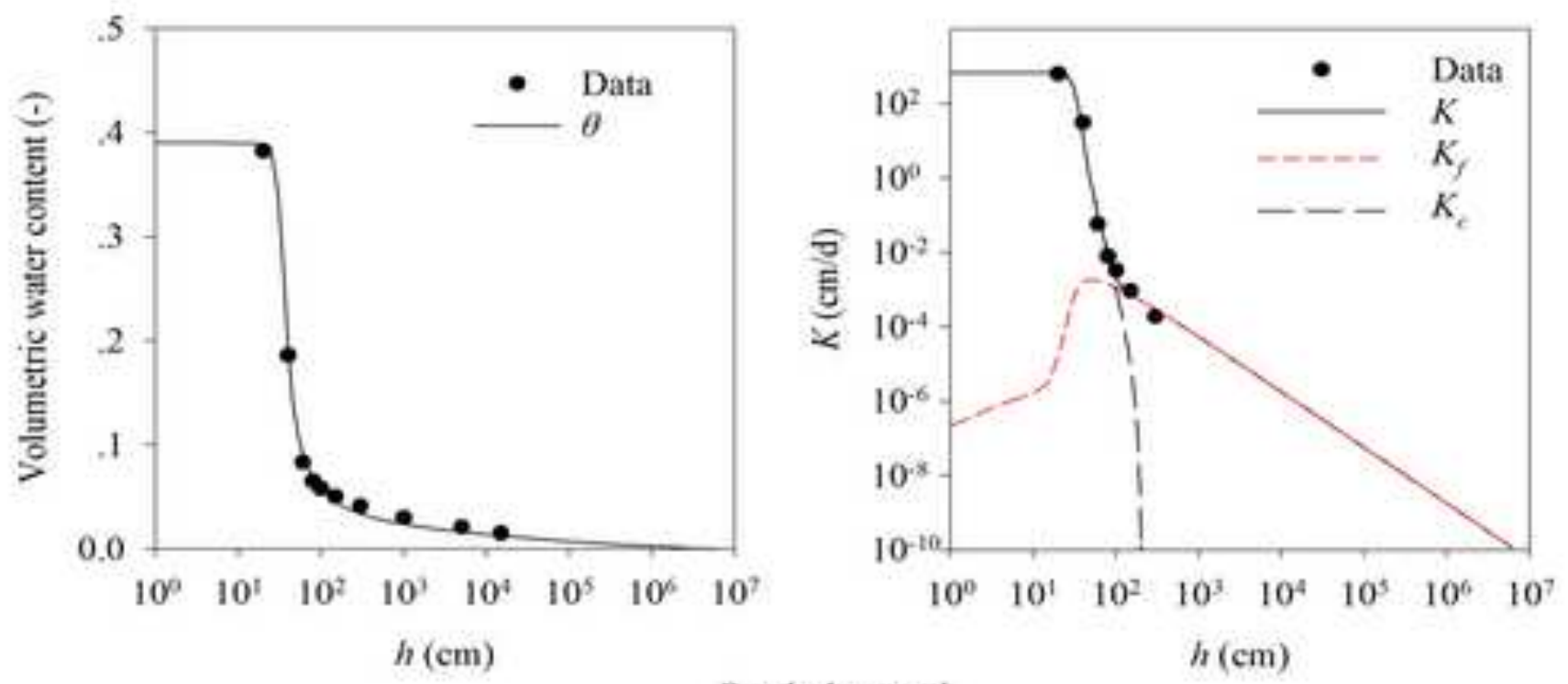

Poederlee sand
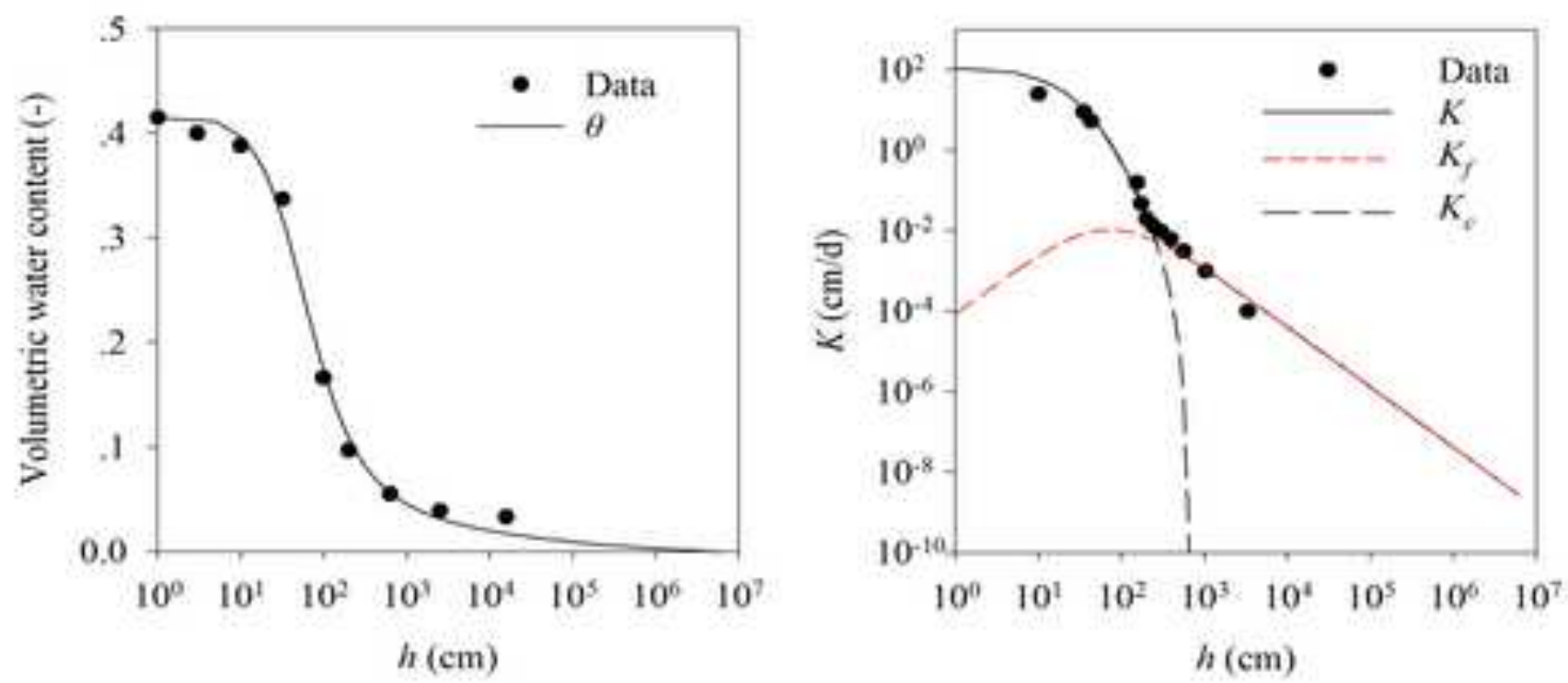
Hupsel sand
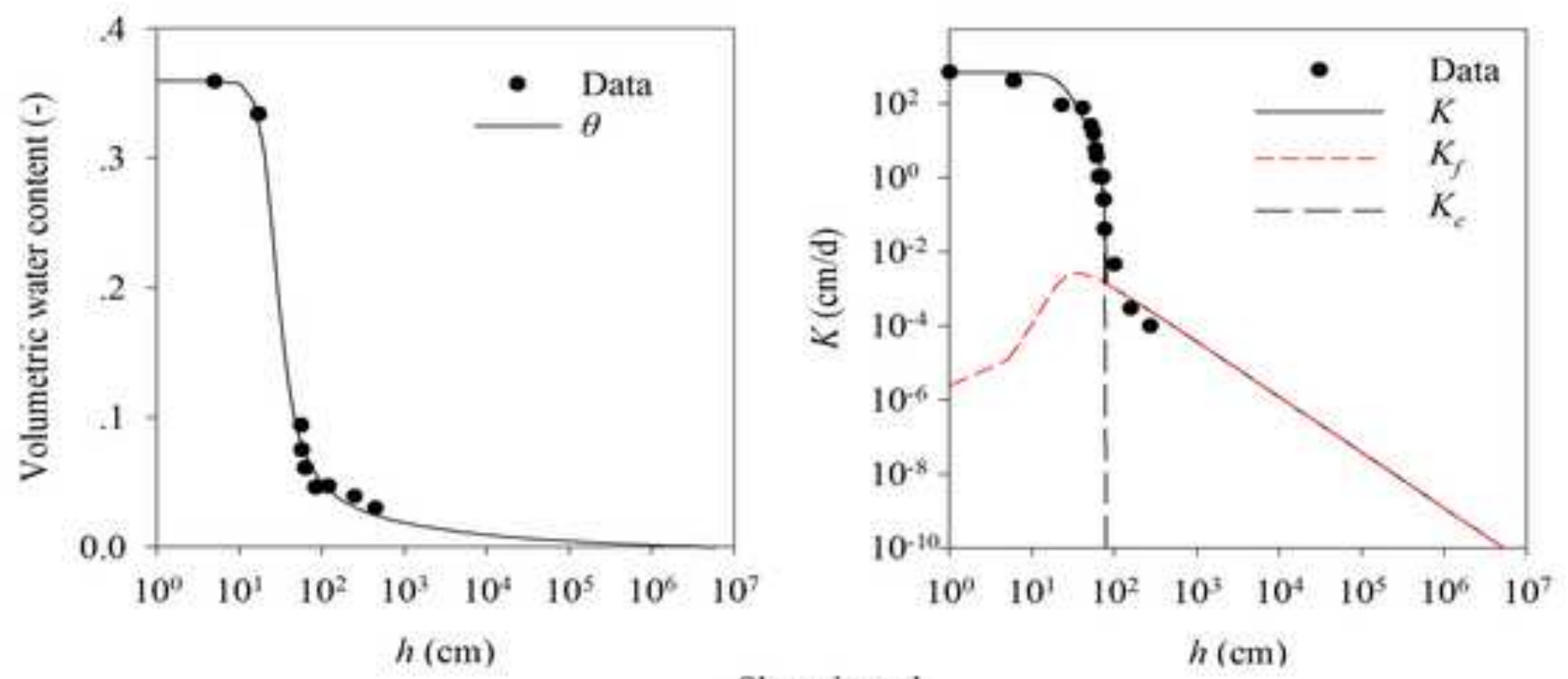

Shonai sand
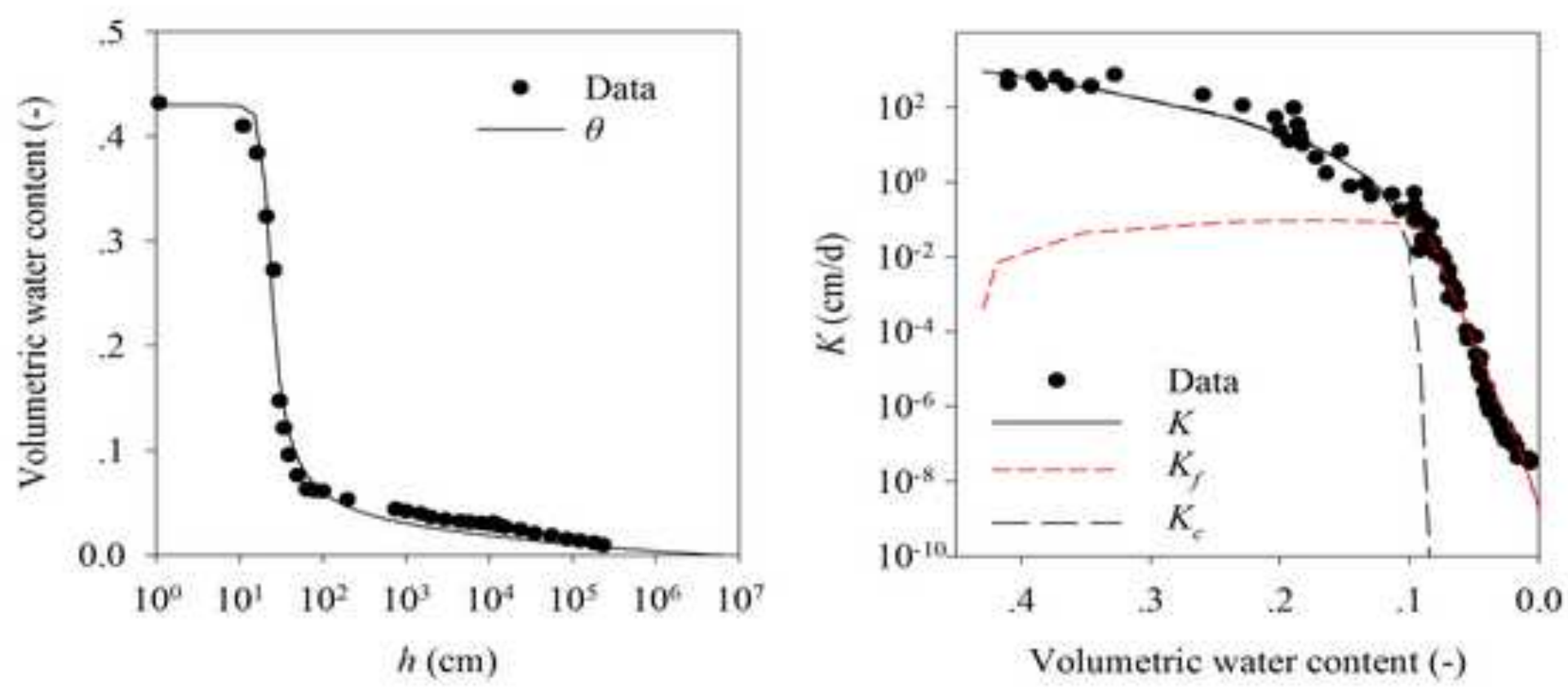
Pachapa fine sandy clay
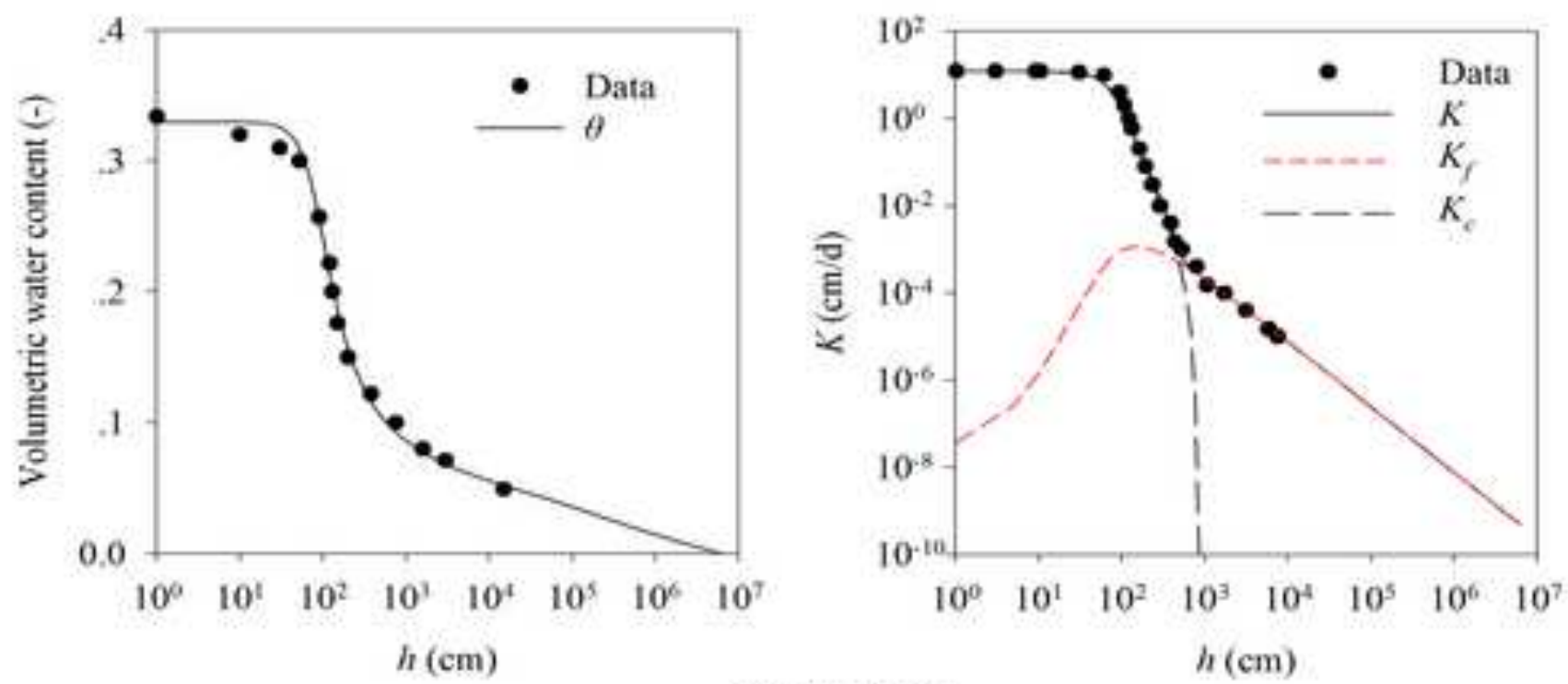

Seelow clay 1
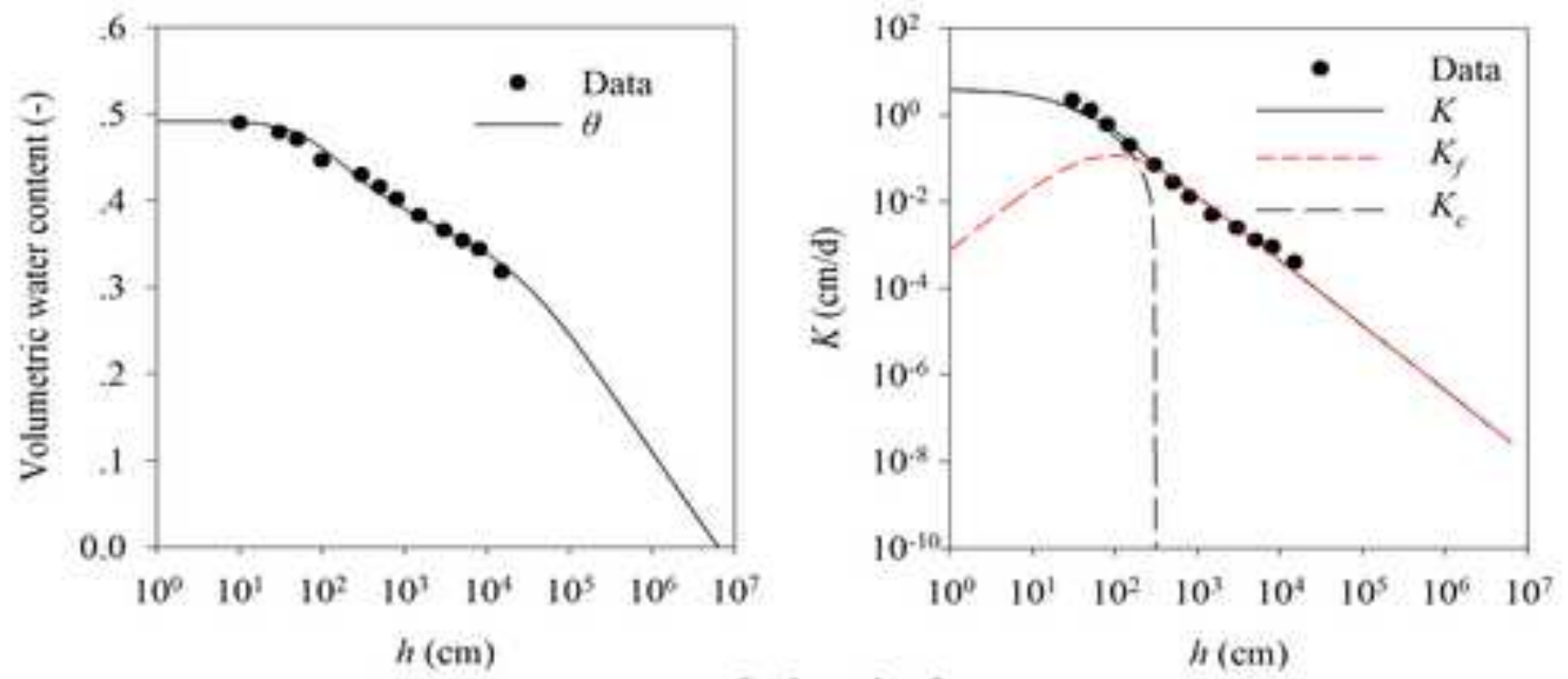

Seelow clay 2
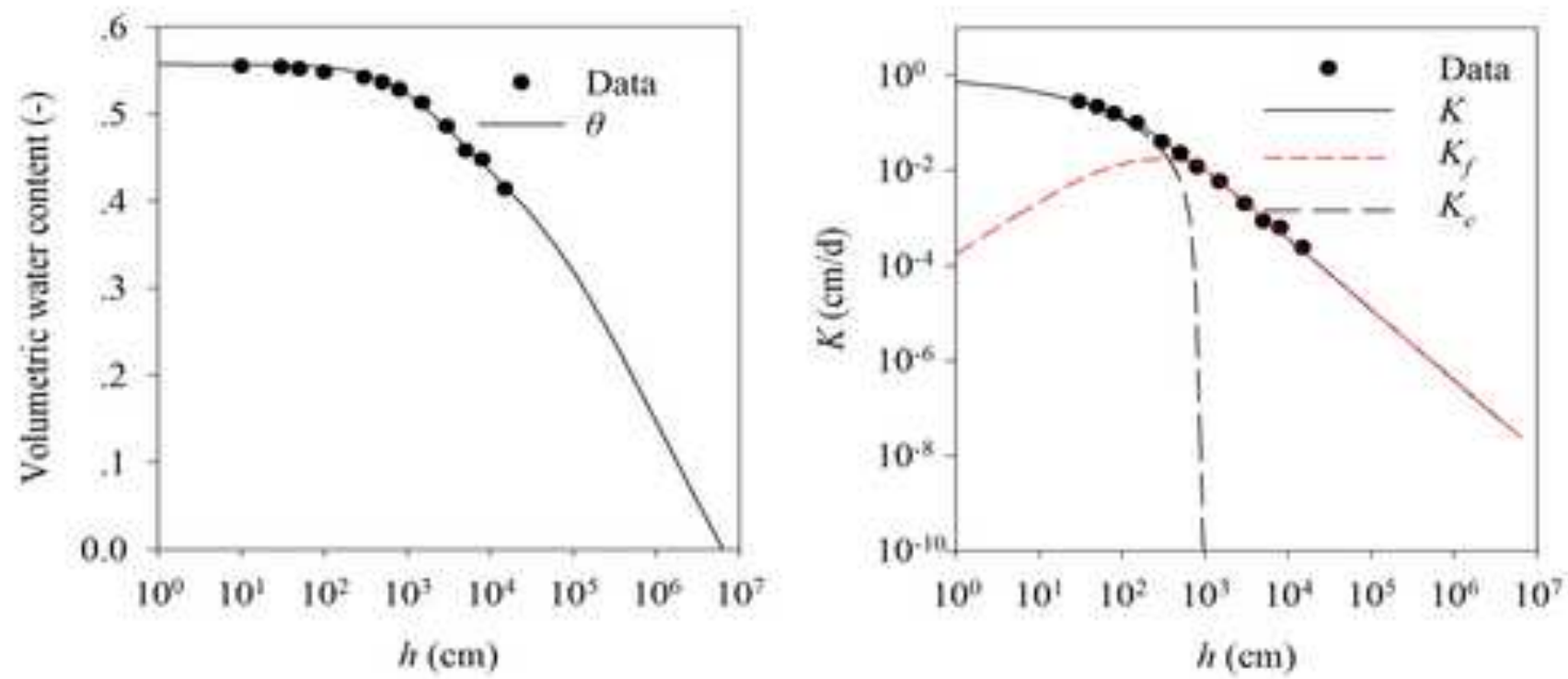
Hollern clay 1
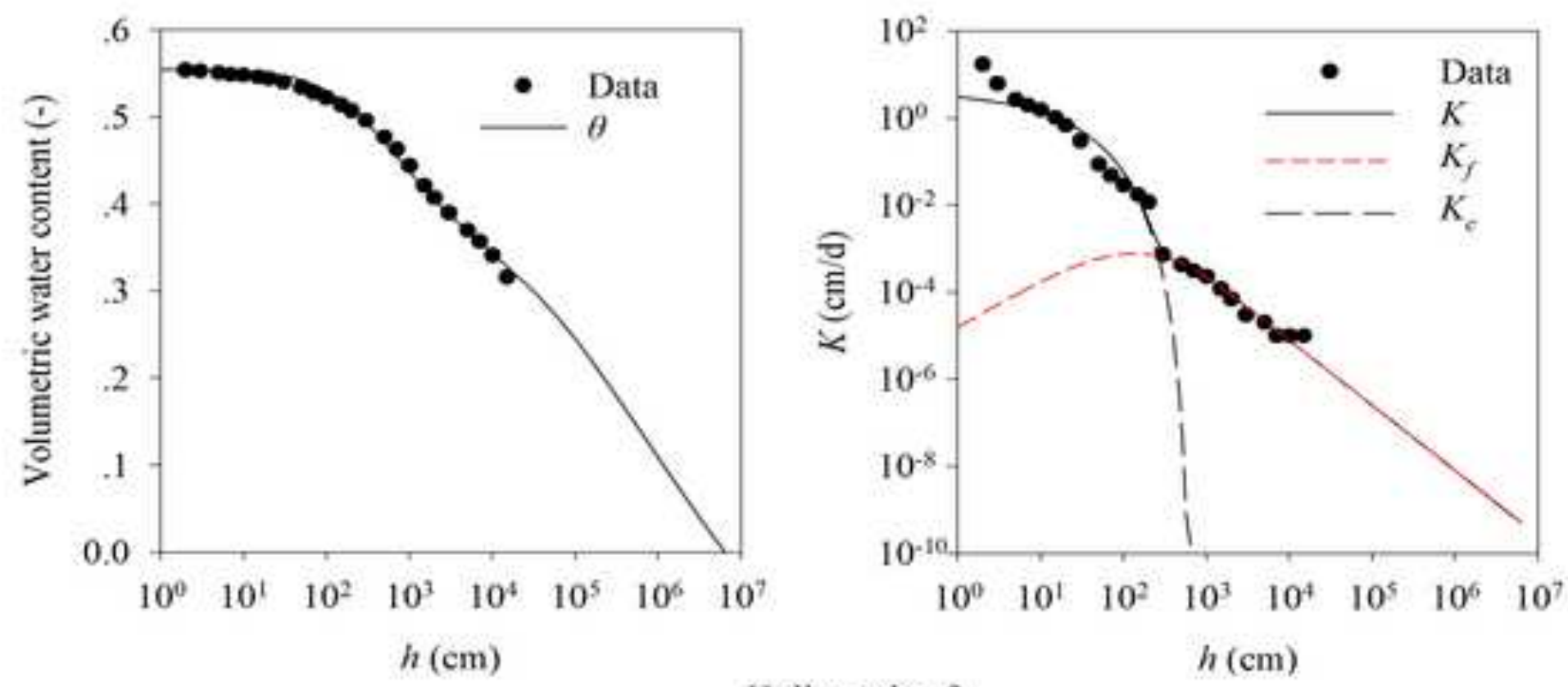

Hollern clay 2
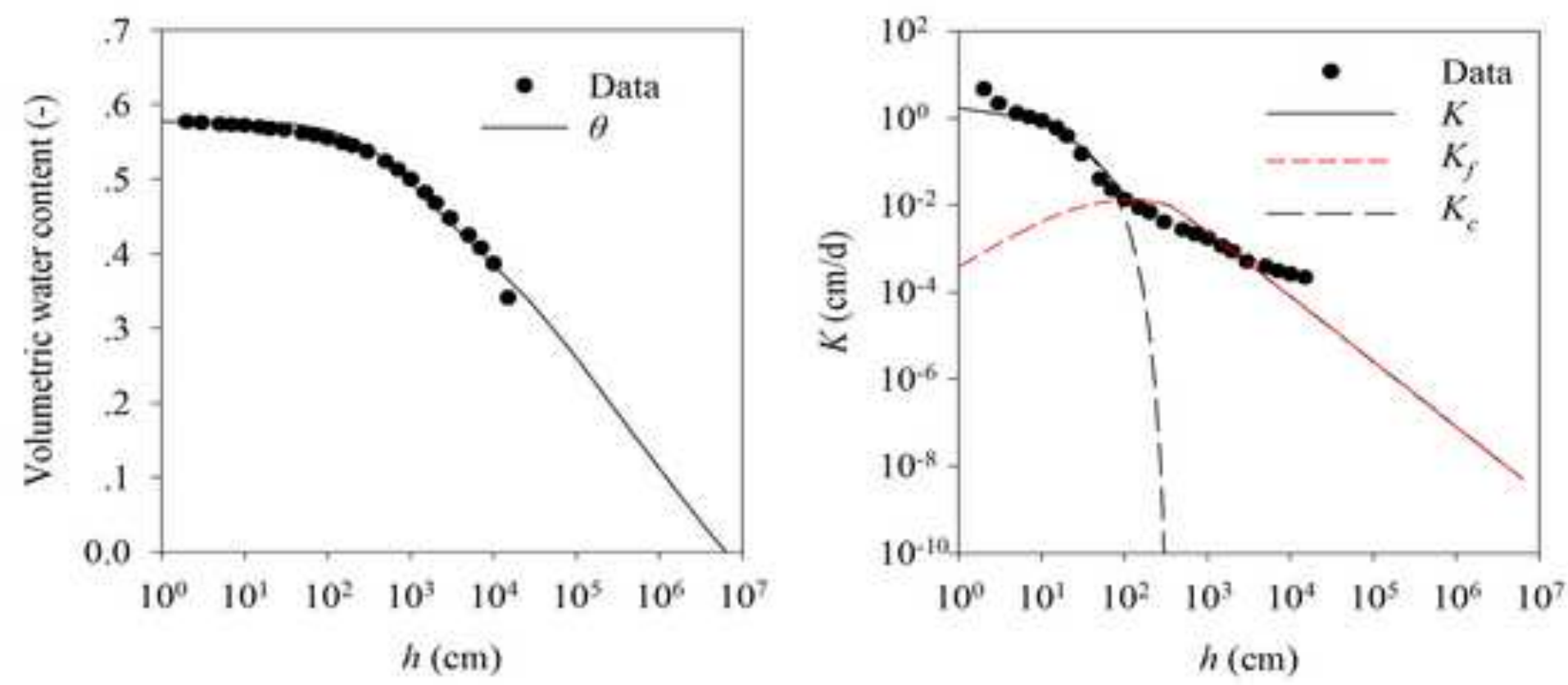


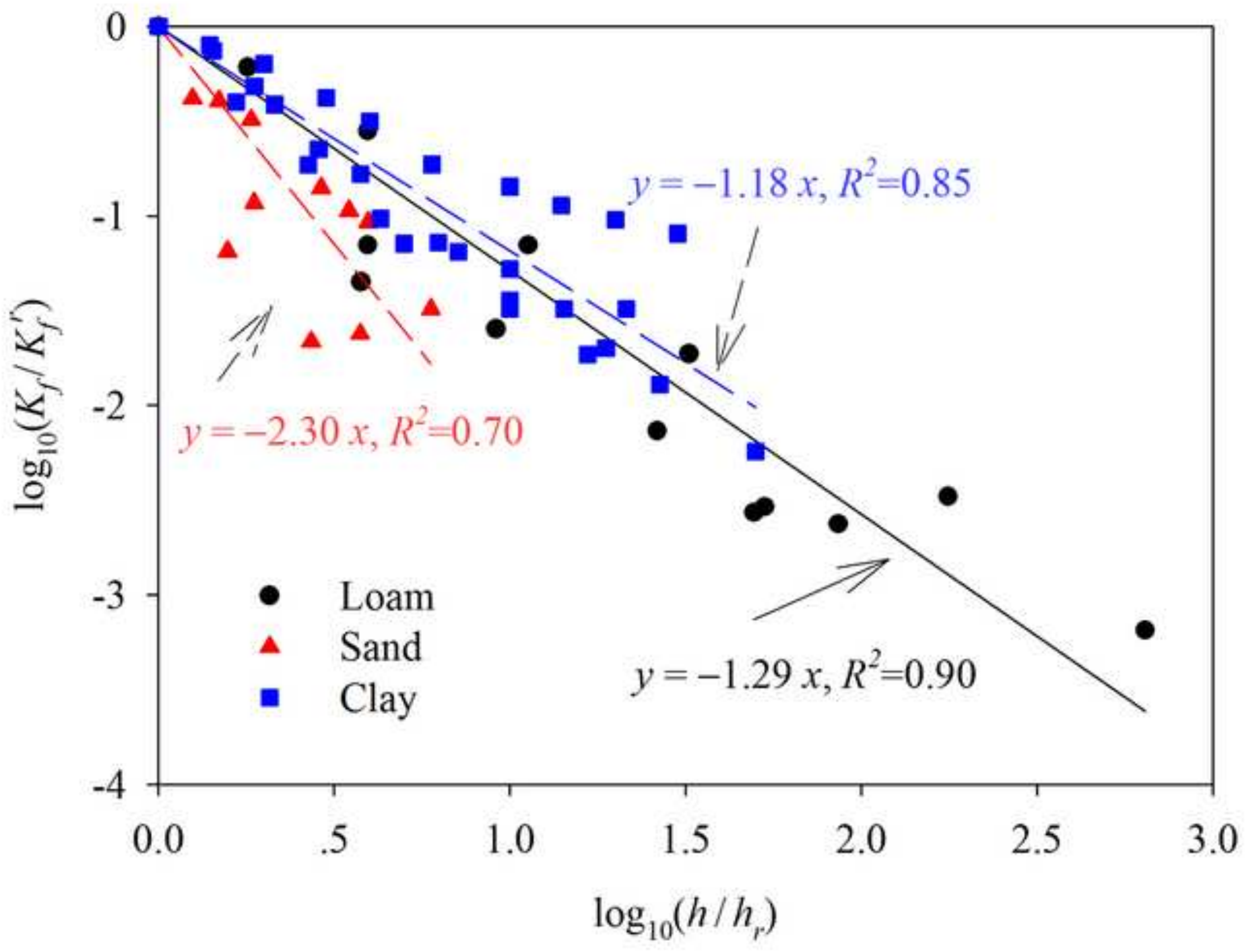



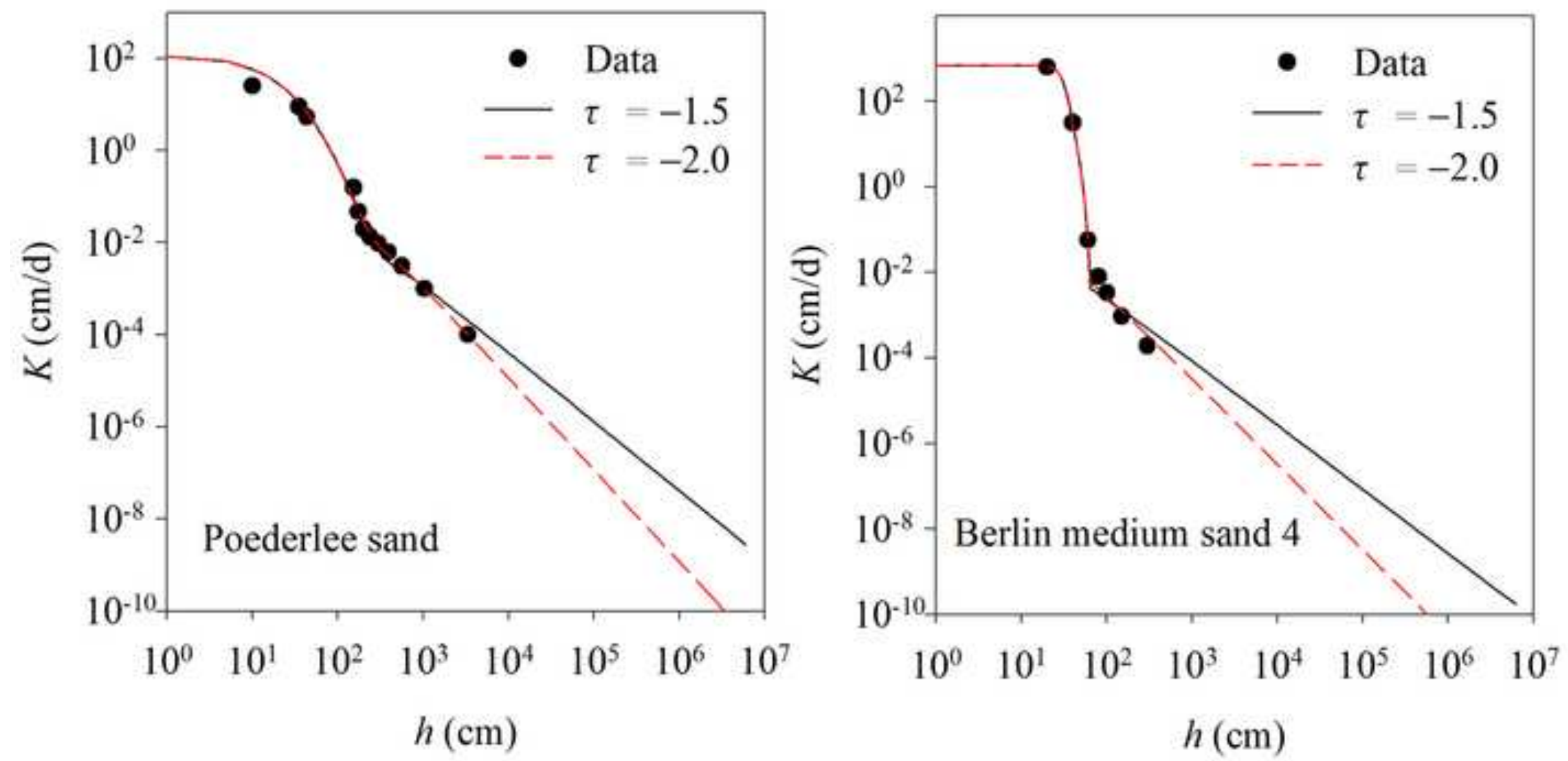
Gilat loam
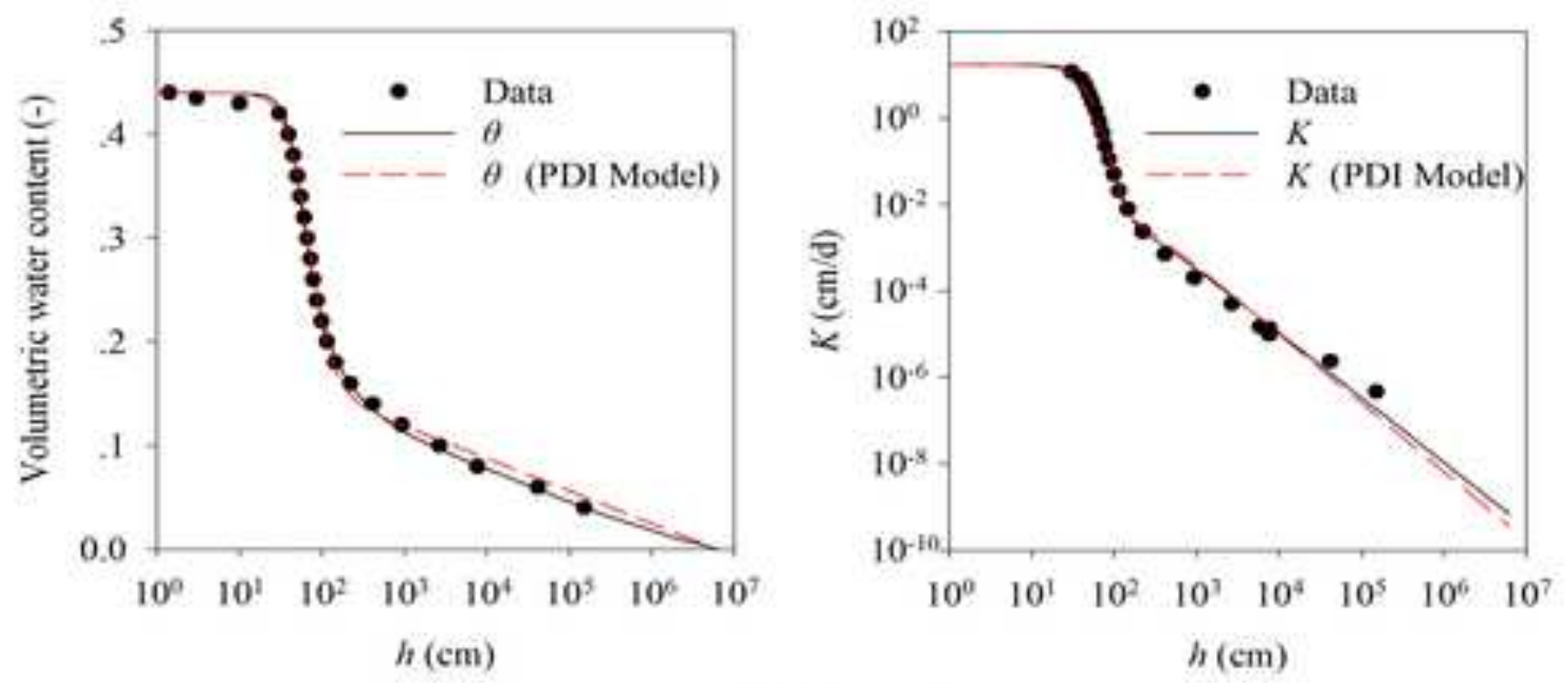

Rehovot sand
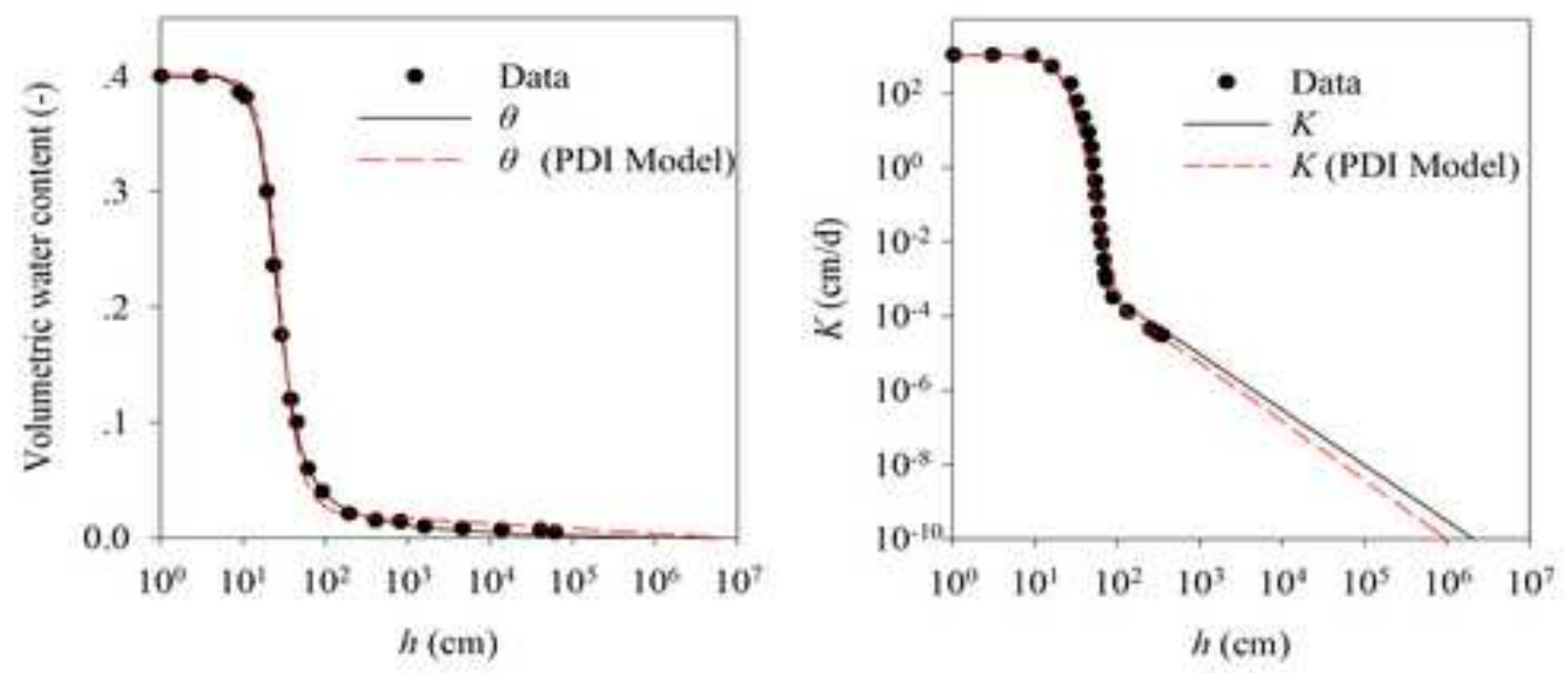

Seelow clay 1
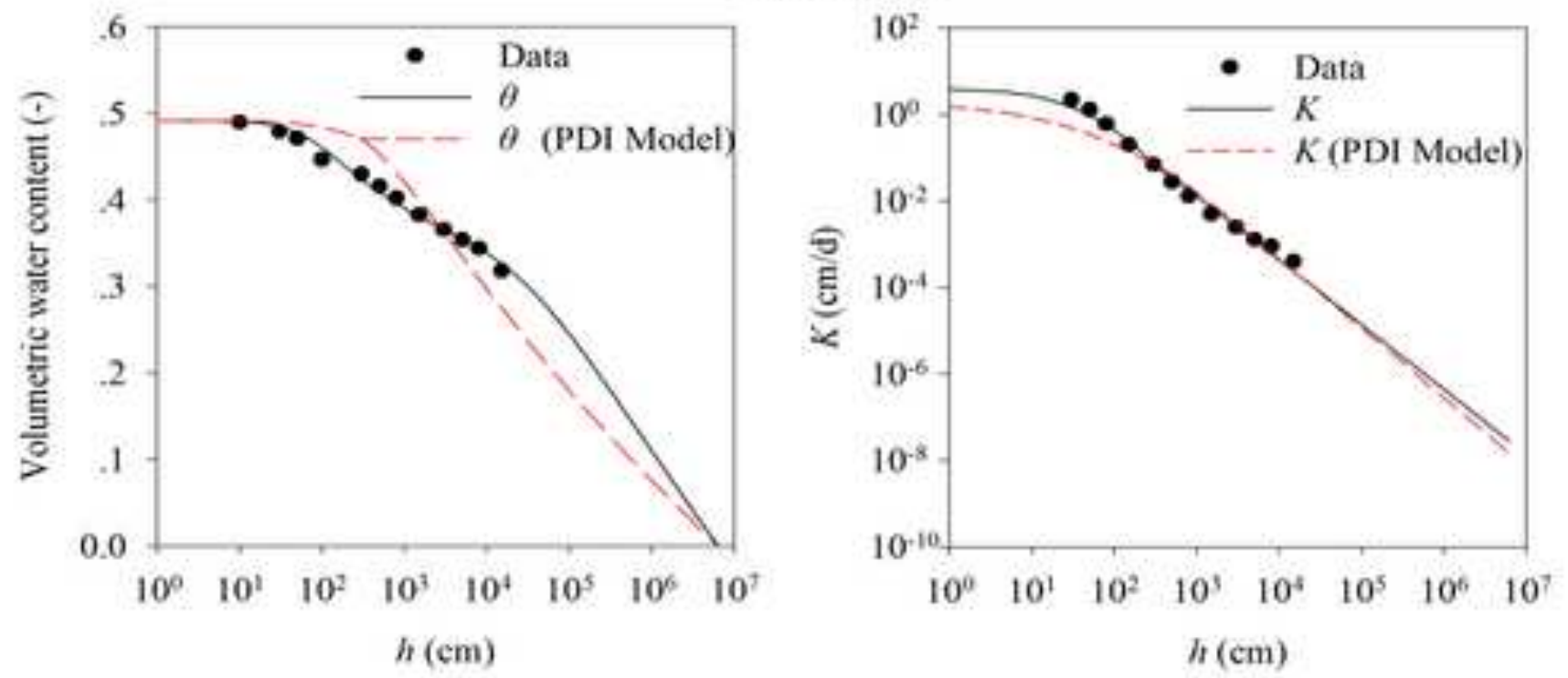
a) $m=0.5$

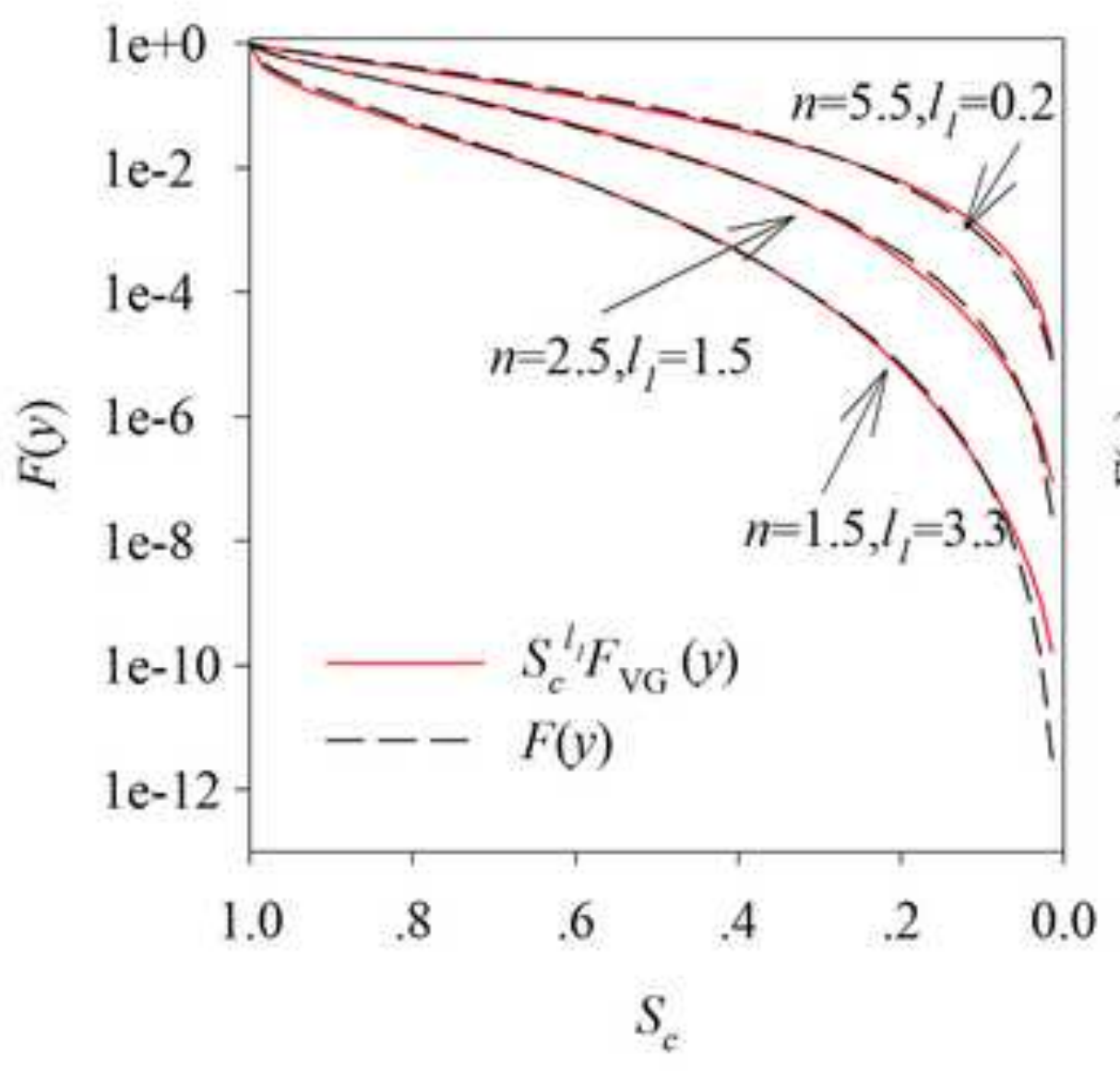

b) $\mathrm{m}=1.5$

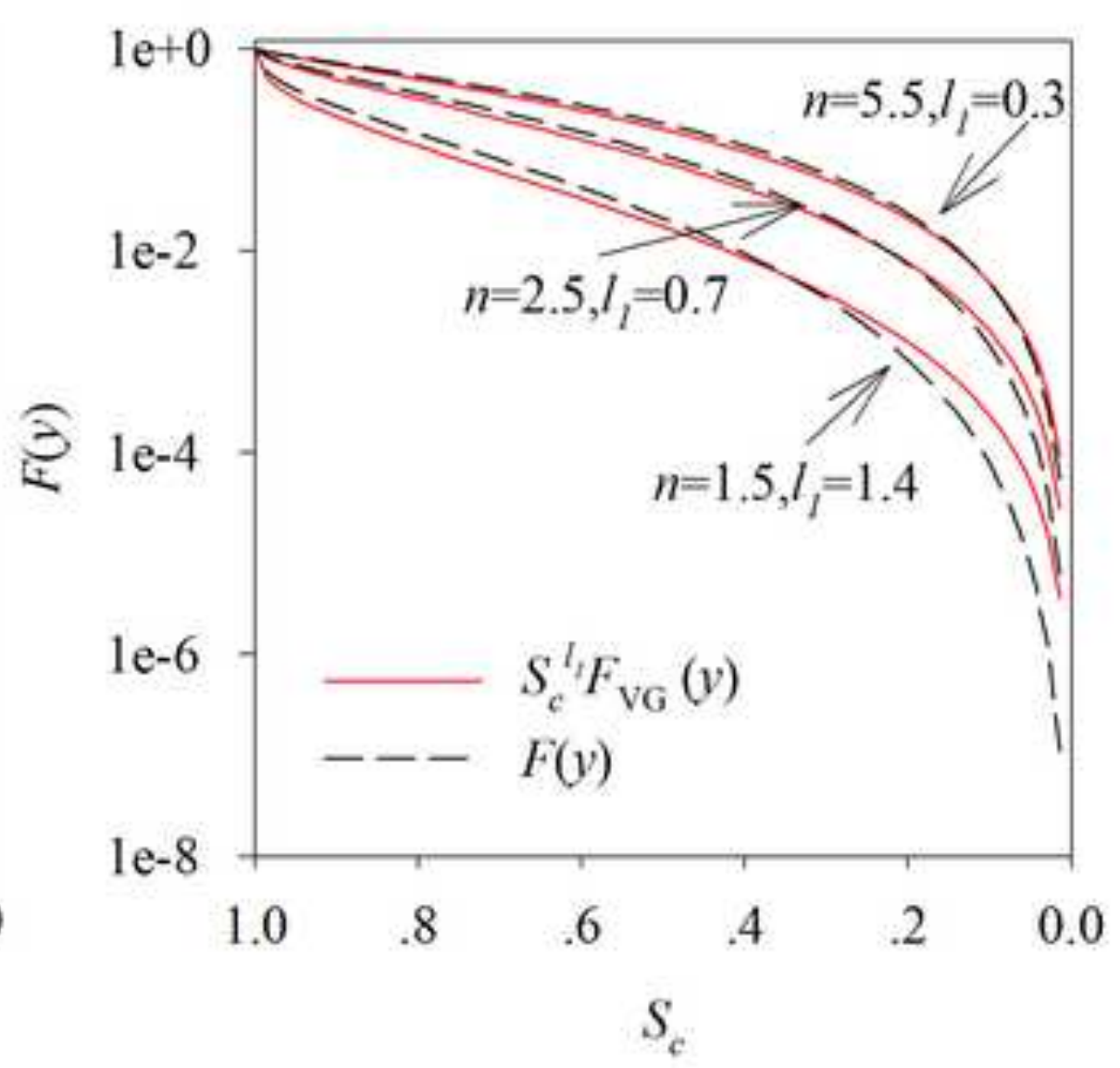




\section{Figure Captions}

Figure 1. Illustration of three different models. No correction means the model without the correction factor (equation (1) with $\mathrm{C}(h)=1$ ), FX model means the model with the correction factor $\mathrm{C}(h)$ (equations (1) and (2)), new model is equations (3) and (4). Parameters set: the values of $h_{r}$ and $m$ are described in title while the other parameters are $n=1.5, \alpha=0.01$.

Figure 2. The model fitting results for Sandy loam and Gilat loam

Figure 3. The model fitting results for Adelanto loam and Pachapa loam

Figure 4. The model fitting results for Poederlee loamy sand and Masa loamy sand

Figure 5. The model fitting results for Rehovot sand, Berlin medium sand 4 and

Poederlee sand

Figure 6. The model fitting results for Hupsel sand and Shonai sand.

Figure 7. The model fitting results for Pachapa fine sandy clay, Seelow clay 1, Seelow clay 2 and Seelow clay 2 .

Figure 8. The model fitting results for Hollern clay 1 and Hollern clay 2.

Figure 9. Log-log plots of the scaled film conductivity vs. scaled suction for different soils.

Figure 10. The model testing results for Poederlee sand and Berlin medium sand 4 with $\tau=-2.0$.

Figure 11. Comparison of the EMFX model results with the PDI model for the Gilat loam, Rehovot sand and Seelow clay 1.

Figure A1. Illustration of the integrated function of the EMFX model, with different $m$ and $n$ values. 